\title{
Automated rapid mapping of joint orientations with mobile LiDAR
}

\author{
Marc J. Gallant ${ }^{\mathrm{a}, *}$, Joshua A. Marshall ${ }^{\mathrm{a}}$ \\ ${ }^{a}$ Mining Systems Laboratory, Queen's University \\ 25 Union Street, Kingston, Ontario, Canada K7L $3 N 6$
}

\begin{abstract}
This paper introduces three-dimensional axis mapping (3DAM), a new method for joint orientation estimation that rapidly collects data from a mobile platform containing a scannerless LiDAR and an inertial measurement unit (IMU). The mobile platform is moved through the environment either as a handheld device or by mounting it to a remotely operated or robotic vehicle. 3DAM is formulated as a global state estimation problem that estimates the orientation of the mobile platform and the joint set orientations by minimizing the uncertainty introduced by the inherently noisy sensors. This requires a dual-parameterization of both the orientation of joint sets and the mobile platform to permit the use of state estimation techniques. 3DAM was field tested in three separate locations and is directly compared to hand measurements and stationary LiDAR. In all experiments, it is shown that 3DAM produces stereonets comparable to other methods, yet does so with lower-cost hardware and significantly reduced effort.
\end{abstract}

Keywords: Joint orientation, Mobile, LiDAR, Laser, Discontinuity, Stereonet

\section{Introduction}

LiDAR has recently been recognized as a valuable tool for rock mass characterization [1]. In particular, there has been considerable interest in using LiDAR for joint orientation estimation [2, 3, 4, 5, 6, 7, 8, 9, 10, 11, 12. Gen5 erally, these methods use a stationary tripod-mounted LiDAR to capture one or more point clouds of the exposed rock face. The orientation of the sensor is measured beforehand such that the collected data can be transformed to the global coordinate frame. Using the geometry of the captured point cloud, the orientations of planar surfaces are extracted and plotted on a stereonet.

10 Joint orientation estimation using LiDAR has several advantages compared to manual techniques (i.e., using a compass and inclinometer). Safety is improved by limiting human exposure to potentially unstable rock faces, it allows

\footnotetext{
* Corresponding author

Email addresses: m.gallant@queensu.ca (Marc J. Gallant), joshua.marshall@queensu.ca (Joshua A. Marshall)
} 
greater access to hard-to-reach locations, it may be faster and less labour intensive, and the opportunity for introducing erroneous measurements due to 15 procedural difficulties, human bias, and human error is significantly reduced. Despite these advantages, there are barriers preventing widespread adoption of stationary LiDAR. Its high cost can be prohibitive, it is often physically too large and heavy for some remote deployments, processing the resulting point clouds often requires manual intervention to remove outliers, and several mea-

20 surements of the same rock face from different points of view are usually required to avoid occlusions. This last drawback can make the data collection process very time-consuming (i.e., moving, reorienting, and taking new measurements with the sensor can take tens of minutes) and is particularly challenging in enclosed spaces (e.g., underground mines).

25 This paper introduces a new algorithm for joint orientation estimation called three-dimensional axis mapping (3DAM) that retains the advantages of using LiDAR measurements for joint orientation estimation, while addressing many of its disadvantages. 3DAM achieves this by using a lightweight, inexpensive mobile platform containing a low-cost, compact three-dimensional LiDAR and

so an inertial measurement unit (IMU). Joint orientations are derived from the collected data by probabilistically determining the orientation of the mobile platform along its trajectory. This is calculated by combining high frequency measurements of the angular velocity of the mobile platform, the direction of gravity, the direction of the Earth's magnetic field, and by repeatedly measuring 35 the orientations of the joint sets themselves.

Many of the disadvantages of using stationary LiDAR are avoided with 3DAM. By capturing a larger number of lower resolution point clouds, the use of a low cost and mobile LiDAR is permitted. Occlusions are easily and quickly eliminated by simply maneuvering around the rock face. The techniques used to

40 process the resulting 3DAM point clouds are fast, fully automated, and require no manual pruning of outliers. The time and effort required to collect data is significantly reduced compared to both manual techniques and stationary LiDAR. Finally, the mobile platform can take any form capable of carrying the sensors, from a handheld configuration to remote operation with a mobile robot.

The focus of this paper is on automatic estimation of joint orientations, with visualization as stereonets. The problems of estimating other characteristics such as, for example, joint spacing, roughness, area/volume estimates, and change detection lie beyond the scope of this work. For these problems, the specific approach developed here may not provide similar advantages.

50 1.1. Related work

Research about joint orientation estimation using LiDAR has focused on different strategies for extracting the orientation of planar surfaces from point clouds. In general, these strategies can be categorized as either top-down or bottom-up approaches. The top-down approach (sometimes called surface re-

55 construction) uses a mesh generated from the point cloud (usually by interpolating between points) to approximate the surface of the measured rock face, and calculates the normal vector at each segment in the mesh. Many researchers 
depend on commercial software (e.g., PolyWorks [13]) to perform surface reconstruction. Slob et al. 14 evaluated this approach and compared it to manual techniques from a cost-benefit perspective. Other researchers have studied optimizing the point cloud processing procedure [5, correcting for biases in data collection [7, and have analyzed the sensitivity to point cloud resolution [6, 10]. The top-down approach often requires manual intervention to prevent unwanted points from being included in the surface reconstruction [10] (e.g., vegetation, outliers).

The bottom-up approach attempts to find subsets of points on planar surfaces and uses least-squares techniques (e.g., principal component analysis) to fit planes to the data. This requires finding subsets of points in the point cloud measuring planar surfaces. A plane is then fit to the subset of points and its 70 normal vector is calculated. Various methods have been used to find the appropriate planar subsets. For example, random sample consensus (RANSAC) has been shown to be an effective way to find subsets belonging to planar surfaces by iteratively comparing subsets to the model of a plane 2, 4, 11. Gigli et al. [9] divide the point cloud into a cubic grid of various resolutions to evaluate the 75 planarity of points in each grid cell. Olariu et al. 3] apply a $k$-means clustering algorithm at different resolutions and measure the planarity of the clusters.

A thorough comparison by Slob [15] of the two techniques concluded that the bottom-up approach is preferable because it is easier to automate, retains the original point cloud, and is better at dealing with outliers. 3DAM uses a so modified form of the bottom-up approach for orientation extraction. Instead of fitting planes to discrete planar surfaces, it estimates the planarity at each point in the point cloud and then clusters similar points across the entire point cloud.

There has been limited research on the use of a mobile platform for joint 85 orientation estimation. De Agostino et al. 16 mounted a GPS, IMU, LiDAR, and camera to a truck, but only captured point clouds while the LiDAR was stationary. As a result, this system was very similar to the established techniques, with the exception that the sensors were more easily moved from one scanning location to the next. A mobile LiDAR system constructed by Terra-

9o point Canada [17. (now Ambercore) and employed by Lato et al. 18] captured point clouds while in motion. This system combines multiple LiDAR sensors, a high-end IMU, and a differential GPS system to track the movement of a truck mounted on railway tracks. The system constructs a massive point cloud in the global coordinate frame, after which a top-down approach was applied to manually selected portions of the point cloud.

Rather than constructing a large, high-resolution point cloud and applying standard techniques to extract the joint orientations, 3DAM is built from the ground up with joint orientation estimation in mind. It is designed to be relatively inexpensive, easily carried and operated by a single person, and 100 have no dependence on GPS. By meeting these constraints, it is deployable in a large variety of environments (i.e., anywhere where a person or remotely operated vehicle can reach, including underground) and is both economically and physically viable as a drop-in replacement for a compass and inclinometer. 
Although the expensive stationary LiDAR devices (i.e., tens of thousands of dollars (1) used in the literature discussed above produce large, high-resolution point clouds - which may be useful for solving problems beyond only joint orientation estimation - they must remain stationary during the data acquisition process that can take tens of minutes per scan. On the other hand, 3DAM uses a low-cost (i.e., hundreds of dollars), lower resolution and mobile LiDAR. The 110 need for high resolution is mitigated by using a LiDAR with a much higher frequency, which captures multiple complete point clouds per second. However, one tradeoff is that this type of low-cost and mobile LiDAR has a shorter range (usually less than 5-10 m) compared with stationary LiDAR (can be tens of metres), making it applicable only in low-range scenarios.

\subsection{About this paper}

This paper begins with mathematical preliminaries used in 3DAM (Section 2p. In particular, background information about the parameterization of joint orientations and state estimation is presented. Next, the 3DAM algorithm is described by decomposing it into smaller sub-algorithms (Section 3), which begins with an overview of the algorithm as a whole (Section 3.1). Information about the field tests is presented (Section 4), followed by a discussion of the experimental results obtained from tests at three different sites (Section 5).

\section{Mathematical preliminaries}

The 3DAM algorithm represents the orientations of planar surfaces in an environment as axes (Section 2.1) and maximizes the likelihood of all measurements using a state estimation algorithm (Section 2.2). Background material about these two topics is presented here.

\subsection{Axes}

An axis is an unordered pair of opposing directions. A phenomenon that is well-represented by an axis is the orientation of a plane in $\mathbb{R}^{3}$. In this example, the unordered pair of opposing directions is the normal vectors on the two sides of the plane. When the planar surfaces are joints, the two degrees of freedom of an axis are often parameterized as dip and dip direction. Although this parameterization is intuitive and useful for visualization purposes, it is not a 135 suitable choice for use in state estimation. This is because dip and dip direction do not form a vector space near the origin. For example, a small perturbation of a near-zero dip can significantly change the dip direction. Furthermore, the dip direction is undefined when dip is zero. Instead, two other parameterizations of axes are used to avoid these issues; namely, unit axes and axis vectors.

${ }_{140}$ A unit axis is an over-parameterization (three parameters, one constraint) of an axis that is free of singularities (i.e., a small perturbation of an axis will always result in a small perturbation of its unit axis parameterization). A unit axis $\mathbf{n}$ is the $3 \times 1$ column

$$
\mathbf{n}:=\left[\begin{array}{l}
\lambda \\
\boldsymbol{\kappa}
\end{array}\right],
$$


where $\lambda \in \mathbb{R}$ and $\boldsymbol{\kappa} \in \mathbb{R}^{2}$ are the scalar and vector parts of the unit axis, respectively. Unit axes are constrained to the unit sphere $S^{2}$; that is, $\lambda^{2}+\boldsymbol{\kappa}^{\top} \boldsymbol{\kappa}=1$. Additionally, $\mathbf{n} \equiv-\mathbf{n}$ because they both represent the same axis. An illustration of the scalar and vector components of a unit axis is shown in Fig.1a Because they are free of singularities, unit axes are a good choice for a global parameterization of axes (i.e., every axis is well-represented by a unit axis).

150 An axis vector is a minimal parameterization (two parameters) of an axis that is well-suited for state estimation problems (e.g., near the origin, axis vectors behave like real vectors). An axis vector $\phi$ is the $2 \times 1$ column

$$
\phi:=\left[\begin{array}{l}
\phi_{1} \\
\phi_{2}
\end{array}\right],
$$

where $\phi_{1}$ and $\phi_{2}$ are scalars. An illustration of the components of an axis vector is shown in Fig.1b. Unlike dip and dip direction, which are also a minimal parameterization of axes, axis vectors behave like vectors in $\mathbb{R}^{2}$ inside the disc in Fig. 1b. In other words, axis vectors can be added together and multiplied by scalars if the result is in the disc. Operations resulting in an axis vector outside of the disc do not satisfy the axioms of a vector space. Fortunately, the nature of the implemented state estimation algorithm allows for the assumption that this will not occur.

Intuitively, axis vectors can be understood as a rotated Cartesian equivalent of dip and dip direction. However, angles in axis vector space are measured counterclockwise (as opposed to clockwise on stereonets). The relationship between an axis vector $\phi=\left(\phi_{1}, \phi_{2}\right)$ and $\operatorname{dip} \alpha$ and dip direction $\beta$ is

$$
\alpha=\sqrt{\phi_{1}^{2}+\phi_{2}^{2}}, \quad \beta=\operatorname{atan} 2\left(\phi_{2}, \phi_{1}\right)+\pi .
$$

Conversions between unit axes and axis vectors are achieved by the following relationships:

$$
\begin{aligned}
\mathbf{n}(\phi) & :=\left[\begin{array}{c}
\cos (\|\phi\|) \\
\operatorname{sinc}(\|\phi\|) \phi
\end{array}\right], \\
\phi(\mathbf{n}) & := \begin{cases}\mathbf{0} & \text { for } \boldsymbol{\kappa}=\mathbf{0} \\
\operatorname{atan} 2(\|\boldsymbol{\kappa}\|, \lambda) \frac{\boldsymbol{\kappa}}{\|\boldsymbol{\kappa}\|} & \text { otherwise }\end{cases}
\end{aligned}
$$

where $\operatorname{sinc}(\phi):=\sin (\phi) / \phi$ and $\operatorname{sinc}(0):=1$. This dual parameterization of axes provides a framework on which state estimation can be performed on joint orientations. Unit axes provide a global parameterization while axis vectors provide a 170 local parameterization that behaves like a vector space under certain conditions. The algorithm described in this paper works with these parameterizations and then converts results to dip and dip direction by applying (3).

\subsection{State estimation}

This section provides the necessary background material to aid understanding of the algorithm described in Section 3. A much more detailed overview of 


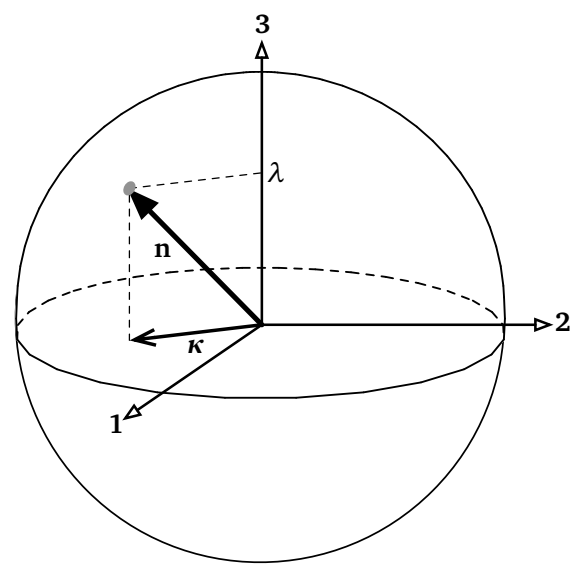

(a) A unit axis $\mathbf{n}$

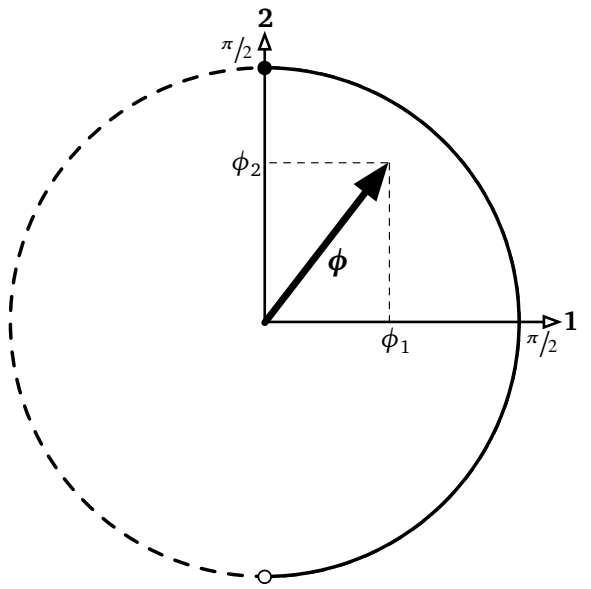

(b) An axis vector $\phi$

Figure 1: Two different parameterizations of axes. State estimation is performed on axes by using unit axes whenever a global singularity-free parameterization is needed, and axis vectors whenever the parameterization needs to behave like a vector space. 
state estimation by Crassidis and Junkins [19, Chapter 1] is recommended to the interested reader. The notation used in this section is based in part on that reference.

A state is a set of variables, called state variables, that describe a dynamical system. For example, the position of a vehicle moving along a one-dimensional road could be the single state variable $x \in \mathbb{R}$. At discrete times, the state trajectory $\mathbf{x}=\left(x_{1}, x_{2}, \ldots, x_{n}\right)$ describes the positions $x_{i} \in \mathbb{R}$ over time. The state is estimated by incorporating noisy measurements (which may involve but not directly measure state variables). For example, at time $k$ the vehicle could 185 count wheel rotations to generate a noisy translation measurement $\tilde{y}_{k}^{(t)} \in \mathbb{R}$, and query its GPS to generate a noisy position estimate $\tilde{y}_{k}^{(p)} \in \mathbb{R}$. It is often practical to model these measurements as Gaussian random variables; i.e.,

$$
\begin{aligned}
& \tilde{y}_{k}^{(t)}=y_{k}^{(t)}+\delta y^{(t)}, \quad \delta y^{(t)} \sim \mathcal{N}\left(0, \sigma_{t}^{2}\right) \\
& \tilde{y}_{k}^{(p)}=y_{k}^{(p)}+\delta y^{(p)}, \quad \delta y^{(p)} \sim \mathcal{N}\left(0, \sigma_{p}^{2}\right)
\end{aligned}
$$

where the true translation $y_{k}^{(t)}$ and true position $y_{k}^{(p)}$ at time $k$ are corrupted by zero-mean Gaussian noises $\delta y^{(t)}$ and $\delta y^{(p)}$ with variances $\sigma_{t}^{2}$ and $\sigma_{p}^{2}$, respectively. 190 This example is illustrated in Fig.2.

Recall that the probability density function (PDF) of a Gaussian random variable $x$ is

$$
f\left(a \mid \mu, \sigma^{2}\right)=\frac{1}{\sigma \sqrt{2 \pi}} \exp \left(-\frac{(a-\mu)^{2}}{2 \sigma^{2}}\right),
$$

which evaluates the likelihood of $x=a$ given the mean $\mu$ and variance $\sigma^{2}$. The likelihood of a measurement is calculated in a similar way, where the mean is 195 the predicted value of the measurement given the state, and the variance is the measurement variance. Returning to our vehicle example, the predicted translation $\mu_{k}^{(t)}$ of a wheel rotation measurement is

$$
\begin{aligned}
\mu_{k}^{(t)} & =x_{k}-x_{k-1} \\
& =\left[\begin{array}{llllll}
0 & \ldots & -1_{k-1} & 1_{k} & \ldots & 0
\end{array}\right]\left[\begin{array}{c}
x_{1} \\
\vdots \\
x_{n}
\end{array}\right] \\
& =\mathbf{F}_{k} \mathbf{x} .
\end{aligned}
$$

Similarly, the predicted position $\mu_{k}^{(p)}$ of a GPS measurement is

$$
\begin{aligned}
\mu_{k}^{(p)} & =x_{k} \\
& =\left[\begin{array}{lllll}
0 & \ldots & 1_{k} & \ldots & 0
\end{array}\right]\left[\begin{array}{c}
x_{1} \\
\vdots \\
x_{n}
\end{array}\right] \\
& =\mathbf{G}_{k} \mathbf{x} .
\end{aligned}
$$




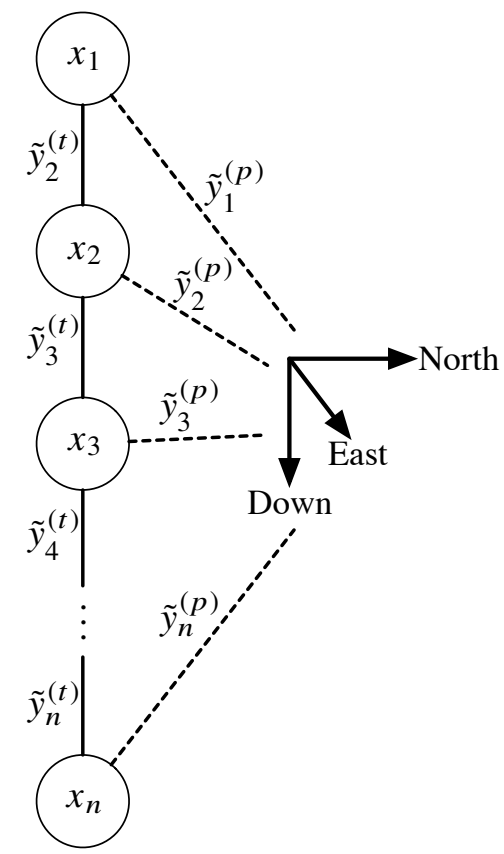

Figure 2: The state trajectory of a one-dimensional vehicle $\mathbf{x}=\left(x_{1}, x_{2}, \ldots, x_{n}\right)$. The translation measurements $\tilde{y}_{k}^{(t)}$ measure the change in position from $x_{k-1}$ to $x_{k}$ and the GPS measurements $\tilde{y}_{k}^{(p)}$ measure the position $x_{k}$ directly (with respect to the global coordinate frame). 
As a result, the likelihoods of the measurements are

$$
\begin{aligned}
f\left(\tilde{y}_{k}^{(t)} \mid \mu_{k}^{(t)}, \sigma_{t}^{2}\right) & =\frac{1}{\sigma_{t} \sqrt{2 \pi}} \exp \left(-\frac{\left(\tilde{y}_{k}^{(t)}-\mathbf{F}_{k} \mathbf{x}\right)^{2}}{2 \sigma_{t}^{2}}\right) \\
f\left(\tilde{y}_{k}^{(p)} \mid \mu_{k}^{(p)}, \sigma_{p}^{2}\right) & =\frac{1}{\sigma_{p} \sqrt{2 \pi}} \exp \left(-\frac{\left(\tilde{y}_{k}^{(p)}-\mathbf{G}_{k} \mathbf{x}\right)^{2}}{2 \sigma_{p}^{2}}\right),
\end{aligned}
$$

200

205

and the negative log-likelihood of the measurements are

$$
\begin{aligned}
& -\ln \left(f\left(\tilde{y}_{k}^{(t)} \mid \mu_{k}^{(t)}, \sigma_{t}^{2}\right)\right)=\eta_{t} \frac{\left(\tilde{y}_{k}^{(t)}-\mathbf{F}_{k} \mathbf{x}\right)^{2}}{2 \sigma_{t}^{2}} \\
& -\ln \left(f\left(\tilde{y}_{k}^{(p)} \mid \mu_{k}^{(p)}, \sigma_{p}^{2}\right)\right)=\eta_{p} \frac{\left(\tilde{y}_{k}^{(p)}-\mathbf{G}_{k} \mathbf{x}\right)^{2}}{2 \sigma_{p}^{2}},
\end{aligned}
$$

where $\eta_{t}$ and $\eta_{p}$ are constants that do not depend on $\mathbf{x}$. The purpose of calculating the negative log-likelihoods becomes clear below. The goal of state estimation is to determine the state estimate that maximizes the likelihood of all the measurements, or equivalently, minimizes the negative log-likelihood of all the measurements.

A common assumption when performing state estimation is that measurements do not influence the outcome of other measurements (i.e., they are independent). As a result, the likelihood of all the measurements is the product of their individual likelihoods, or equivalently, the sum of their individual negative log-likelihoods. This allows for the creation of a cost function $J(\mathbf{x})$ to be minimized. For our vehicle example, the cost function is

$$
J(\mathbf{x})=\sum_{k=2}^{n} \frac{\left(\tilde{y}_{k}^{(t)}-\mathbf{F}_{k} \mathbf{x}\right)^{2}}{2 \sigma_{t}^{2}}+\sum_{k=1}^{n} \frac{\left(\tilde{y}_{k}^{(p)}-\mathbf{G}_{k} \mathbf{x}\right)^{2}}{2 \sigma_{p}^{2}},
$$

where the constants $\eta_{t}$ and $\eta_{p}$ are not included because they do not depend on the state. The difference between the actual and the predicted measurements is termed the residual error $e(\mathbf{x})$; e.g.,

$$
e_{k}^{(t)}(\mathbf{x})=\tilde{y}_{k}^{(t)}-\mathbf{F}_{k} \mathbf{x} \quad \text { and } \quad e_{k}^{(p)}(\mathbf{x})=\tilde{y}_{k}^{(p)}-\mathbf{G}_{k} \mathbf{x}
$$

are the translation and position residual errors, respectively. Note that the errors are linear with respect to the state. As a result, because the cost function no longer involves exponentials as a consequence of using the negative log-likelihood of the measurements, it is quadratic with respect to the state trajectory. This makes minimizing the cost function straightforward; its minimum is obtained by setting its derivative with respect to $\mathbf{x}$ to zero and solving for $\mathbf{x}$. 
Although the principles are the same, this paper deals with a much more difficult state estimation problem then our vehicle example. First, the state consists of the orientation of the mobile platform and the axes of the joint sets. Both of these require global parameterizations to avoid singularities and local parameterizations to behave like vector spaces. Next, the residual errors are nonlinear with respect to the state. As a result, the cost function is linearized and is no longer quadratic in the state; rather, it is quadratic in a perturbation of the state. This makes finding the optimal state trajectory iterative; optimal perturbations are found and applied to the state until convergence criteria are

230 met. Finally, when the axis of a planar surface is measured, it is not known to which joint set it belongs. This matching of measurements with joint sets is called data association and can result in a poor state estimate if performed incorrectly. Despite these differences, the algorithm outlined in Section 3 is structured similarly to the simplified example presented in this section.

\section{Three-dimensional axis mapping}

Three-dimensional axis mapping (3DAM) is a state estimation algorithm in the space of orientations and axes. The state trajectory $\mathbf{x}$ to be estimated in $3 \mathrm{DAM}$ is

$$
\mathbf{x}=(\underbrace{\mathbf{q}_{1}, \mathbf{q}_{2}, \ldots, \mathbf{q}_{n}}_{\mathbf{Q}}, \underbrace{\mathbf{n}_{1}, \mathbf{n}_{2}, \ldots, \mathbf{n}_{m}}_{\mathbf{A}}),
$$

where $\mathbf{q}_{i}$ represents the orientation of a mobile platform at different points in its trajectory, and $\mathbf{n}_{j}$ represents the axis of a joint set. The sequence of mobile platform orientations $\mathbf{Q}$ represent all of the orientations at which a LiDAR measurement was taken, and the axis map $\mathbf{A}$ is a list of all the joint sets present in the environment of interest. Note that $\mathbf{Q}$ and $\mathbf{A}$ contain no positional information. For the same reason the two parameterizations of axes were introduced in 245 Section 2.1. a global and minimal parameterization of orientations is required. 3 DAM uses unit quaternions [20] as the global parameterization and rotation vectors 21] as the minimal parameterization.

One might question why the state trajectory includes $\mathbf{Q}$, when $\mathbf{A}$ contains the joint set information. This is a the result of the chicken-and-egg problem known as simultaneous localization and mapping (SLAM) 22 in the robotics community. To be able to build a map (in this case, a stereonet), knowledge of the position of the sensors is required (in this case, the orientation of the mobile platform). Conversely, knowledge of the position of the sensors in the map requires the map itself. As a result, both are estimated simultaneously.

\subsection{Overview}

This section provides a high-level overview of how 3DAM works. Before describing each step in greater detail in the subsequent sections, the steps involved in the algorithm are enumerated below. 
(i) Data collection (Section 3.2, Fig. 3). A mobile platform is equipped with a $3 \mathrm{D} \mathrm{LiDAR}$ and an inertial measurement unit (IMU). It is moved through the environment such that the field of view of the LiDAR captures all the surfaces to be mapped.

(ii) Orientation measurements (Section 3.3). Measurements of the orientation of the mobile platform are calculated using the IMU data. An initial estimate of $\mathbf{Q}$ is derived from these measurements.

(iii) Axis measurements (Section 3.4, Fig.4, Fig.5). Measurements of the axes of planar surfaces are extracted from each LiDAR measurement. The axes are rotated into the global coordinate frame using the initial estimate of $\mathbf{Q}$ and are clustered into joint sets, forming an initial estimate of $\mathbf{A}$.

(iv) Building the cost function (Section 3.5, Fig. 6). The negative log-likelihoods of the LiDAR axis measurements and the IMU orientation measurements are assembled into a cost function.

(v) Minimizing the cost function (Section 3.6). The cost function is minimized, resulting in a perturbation of the state trajectory $\Delta \mathbf{x}$. If this perturbation is large enough and applying it to the state trajectory reduces the cost, the cost function is rebuilt and minimized again.

(vi) Generating the stereonet (Section 3.7, Fig.7). All axis measurements are rotated into the global coordinate frame using the now-optimized estimate of $\mathbf{Q}$. The axes are then converted to dip and dip direction using (3) to generate a stereonet.

In steps (iii) and (iii) above, an initial estimate of the state trajectory $\mathbf{x}=$ $(\mathbf{Q}, \mathbf{A})$ is calculated. The state estimation example in Section 2.2 does not require an initial estimate because the residual errors in $\sqrt{12}$ are linear with respect to the state. This results in a quadratic cost function that is easily minimized. However, the analogous residual errors in 3DAM are nonlinear. As a result, the residual errors are linearized about an initial estimate of $\mathbf{x}$ using a first order Taylor series expansion [23, Chapter 11]; i.e.,

$$
e(\mathbf{x}) \approx e(\hat{\mathbf{x}})+\left.\frac{\partial e(\mathbf{x})}{\partial \mathbf{x}}\right|_{\hat{\mathbf{x}}} \Delta \mathbf{x},
$$

where $\hat{\mathbf{x}}$ is an initial estimate of $\mathbf{x}$ and $\Delta \mathbf{x}$ is a perturbation of the initial estimate. Calculating the residual errors using (14) makes the cost function quadratic in $\Delta \mathbf{x}$ and requires an initial estimate of the state trajectory $\hat{\mathbf{x}}=$ $(\hat{\mathbf{Q}}, \hat{\mathbf{A}})$.

\subsection{Data collection}

Data is collected with a 3D LiDAR and an inertial measurement unit (IMU). These two sensors must be rigidly connected such that their relative orientation is constant and known. The mobile platform containing these sensors is 


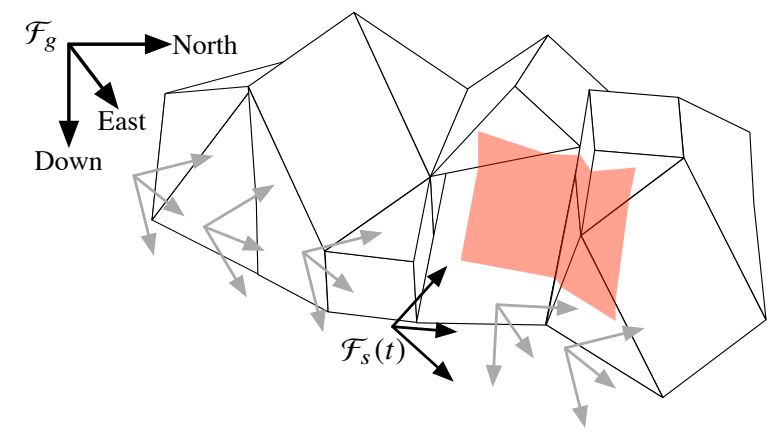

Figure 3: The trajectory of the mobile platform is represented by its coordinate frame $\mathcal{F}_{s}$, whose orientation changes over time with respect to the global coordinate frame $\mathcal{F}_{g}$. A LiDAR measurement at one point along the trajectory is shown. LiDAR measurements are taken until all desired surfaces have been measured, where each LiDAR measurement captures a portion of the rock face.

moved through the environment until all planar surfaces to be mapped have been scanned by the LiDAR. Note that different types of locomotion can be employed (e.g., handheld, wheeled robot, flying robot). All measurements made by the mobile platform are expressed in the sensor coordinate frame $\mathcal{F}_{s}$, whose orientation with respect to the global coordinate frame $\mathcal{F}_{g}$ (North-East-Down) changes over time. This relationship is illustrated in Fig. 3 .

The LiDAR measurements are point clouds expressed in $\mathcal{F}_{s}$. It is assumed that the position and orientation of the mobile platform is the same for all points measured by the LiDAR in a single point cloud. This assumption can be met using one of two strategies: (i) ensure that the mobile platform is stationary during a LiDAR measurement, or (ii) use a LiDAR that captures all points simultaneously. The second strategy is used in the experiments in this paper; however, the first strategy is also compatible with 3DAM but may restrict the form of locomotion of the mobile platform.

The IMU consists of a three-dimensional accelerometer, a three-dimensional gyroscope, and a three-dimensional magnetometer. The accelerometer is used to measure acceleration due to gravity in $\mathcal{F}_{s}$, whose direction in the $\mathcal{F}_{g}$ is known (down). As a result, non-gravitational acceleration of the mobile platform should be avoided; however, the uncertainty introduced by non-gravitational acceleration is accounted for in the state estimation algorithm. The gyroscope is used to measure the angular velocity of the mobile platform in $\mathcal{F}_{s}$, and the magnetometer is used to measure the direction of the Earth's magnetic field in $\mathcal{F}_{s}$, whose direction in $\mathcal{F}_{g}$ is known (magnetic north).

\subsection{Orientation measurements}

320

A Kalman filter (KF) [24] is used to transform the IMU data into measurements of the orientation of the mobile platform and to calculate $\hat{\mathbf{Q}}$. A KF uses a sequence of noisy sensor measurements (or command inputs) to probabilistically determine the maximum a posteriori estimate of an unknown state 
(i.e., the most likely state after resolving the measurements). The KF implementation in 3DAM measures changes in orientation of the mobile platform by integrating gyroscope measurements, and directly infers the orientation by comparing the accelerometer and magnetometer measurements with the known direction of gravity and the Earth's magnetic field.

The result of the KF is an orientation measurement $\tilde{\mathbf{y}}^{(o)}$ of the mobile platform at each LiDAR measurement. These measurements are modelled as

$$
\tilde{\mathbf{y}}^{(o)}=\mathbf{y}^{(o)} \boxplus \delta \mathbf{y}^{(o)}, \quad \delta \mathbf{y}^{(o)} \sim \mathcal{N}\left(0, \mathbf{R}_{o}\right),
$$

where the true orientation $\mathbf{y}^{(o)}$ is corrupted by zero-mean noise $\delta \mathbf{y}^{(o)}$ with covariance matrix $\mathbf{R}_{o}$. Both $\tilde{\mathbf{y}}^{(o)}$ and $\mathbf{y}^{(o)}$ are global parameterizations of orientations (i.e., they are singularity free), while $\delta \mathbf{y}^{(o)}$ is a minimal parameterization (i.e., it behaves like a vector). The $\boxplus$ operator is used in place of addition to signify 335 that $\delta \mathbf{y}^{(o)}$ must be converted to the global parameterization before perturbing $\mathbf{y}^{(o)}$. The noise is minimally parameterized so that $\mathbf{R}_{o}$ properly reports uncertainty only about the (three) degrees of freedom of an orientation (i.e., $\mathbf{R}_{o}$ is a $3 \times 3$ matrix) .

The initial orientation estimate is simply $\hat{\mathbf{Q}}=\left(\tilde{\mathbf{y}}_{1}^{(o)}, \tilde{\mathbf{y}}_{2}^{(o)}, \ldots, \tilde{\mathbf{y}}_{n}^{(o)}\right)$. In other words, $\mathbf{Q}$ is initially estimated using only the IMU measurements. It is later optimized by minimizing the cost function that involves both the orientation measurements derived from the IMU data (this section) and axis measurements from the LiDAR data (Section 3.4).

\subsection{Axis measurements}

The point cloud generated by a LiDAR measurement is reduced to a small number of axis measurements. Each axis measurement represents a dominant, reoccurring axis observed in a point cloud. Axis extraction is performed in two steps: calculation of point axes (the axis of the surface measured at each point in the point cloud) and grouping similar point axes into clusters. A summary of axis extraction is illustrated in Fig. 4 and is described in detail below.

The axis (normal vector) of each point in the point cloud is estimated using a robust normal estimation algorithm [25]. A plane is fit to a subset of the points in the local neighbourhood of each point using principal component analysis (PCA) 26]. The algorithm only selects points in the neighbourhood meeting consistency and orthogonal distance criteria. PCA also quantifies the planarity of the neighbourhood of points, and points determined to be on non-planar surfaces are discarded.

The large set of point axes is clustered to remove outliers and to produce a single axis measurement from each cluster. An unsupervised clustering al360 gorithm (DBSCAN [27]) that clusters only dense concentrations of point axes is used for this purpose. DBSCAN automatically determines an appropriate number of clusters and marks point axes as outliers in regions of low density. Point axes belonging to the same joint set (but not necessarily belonging to the same plane) appear as clusters in axis vector space. The mean and covariance

of each cluster form the axis measurements. Note that one cannot simply take 


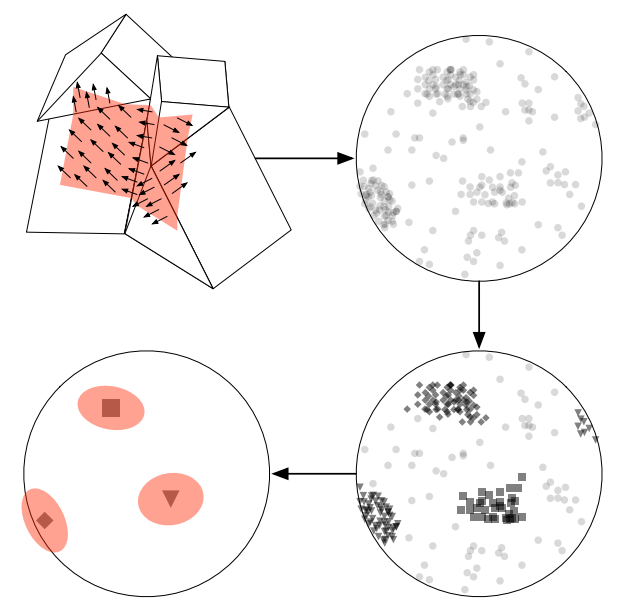

Figure 4: Generating a small number of axis measurements from a point cloud. First, the axis of each point is estimated (top left) and is transformed into a large set of axis vectors (top right). Next, DBSCAN clusters similar axes and ignores outliers (bottom right). Finally, the axial mean and covariance of each cluster is calculated, which form the axis measurements (bottom left). The uncertainties of the axis measurements (due to the spread in the clusters) are illustrated as error ellipses.

the arithmetic mean and covariance of a set of axes; rather the axial mean and axial covariance is calculated 28 .

Each cluster produces an axis measurement $\tilde{\mathbf{y}}^{(a)}$ that is modelled as

$$
\tilde{\mathbf{y}}^{(a)}=\mathbf{y}^{(a)} \boxplus \delta \mathbf{y}^{(a)}, \quad \delta \mathbf{y}^{(a)} \sim \mathcal{N}\left(0, \mathbf{R}_{a}\right),
$$

where the true axis $\mathbf{y}^{(a)}$ is corrupted by zero-mean noise $\delta \mathbf{y}^{(a)}$ with covariance matrix $\mathbf{R}_{a}$. Both $\tilde{\mathbf{y}}^{(a)}$ and $\mathbf{y}^{(a)}$ are parameterized as unit axes, while $\delta \mathbf{y}^{(a)}$ is parameterized as an axis vector. As is the case in (15), the $\boxplus$ operator is used to combine the global and minimal parameterizations, and the covariance matrix $\mathbf{R}_{a}$ represents the uncertainty only about the degrees of freedom (i.e., $\mathbf{R}_{a}$ is a $2 \times 2$ matrix). This paper uses error ellipses to illustrate the uncertainty represented by $\mathbf{R}_{a}$, such as those in Fig.4

At this point, each LiDAR measurement has been reduced to a small number of axis measurements. However, it is not yet known if axis measurements originating from different point clouds measure the same joint set. Because each LiDAR measurement is taken at a different orientation of the mobile platform, 380 the axis measurements must be rotated to a common coordinate frame to be compared. Fortunately, all axis measurements can be rotated to $\mathcal{F}_{g}$ using the estimated orientations in $\hat{\mathbf{Q}}$. In this common coordinate frame, the axes of planar surfaces belonging to joint sets appear as clusters of axis measurements. The axial mean of each of these clusters form the initial axis map estimate $\hat{\mathbf{A}}$. This 385 process is illustrated in Fig. 5 . The entry of $\hat{\mathbf{A}}$ to which each axis measurement belongs (i.e., it was part of the joint set cluster) is recorded, and outliers (i.e., axis measurements not belonging to a cluster) are ignored. 


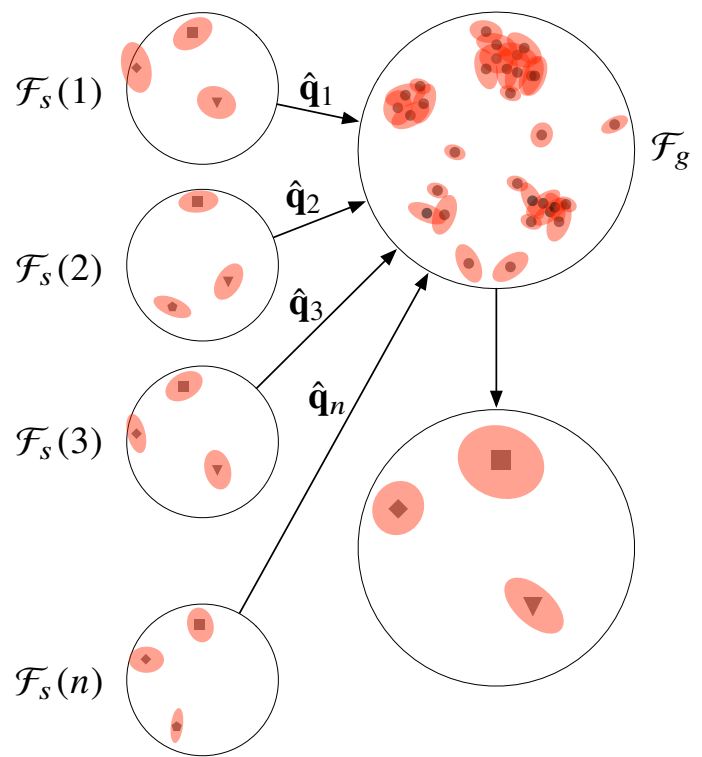

Figure 5: Axis measurements are expressed in $\mathcal{F}_{S}(t)$, the coordinate frame of the mobile platform at time $t$. Using $\hat{\mathbf{Q}}=\left(\hat{\mathbf{q}}_{1}, \ldots \hat{\mathbf{q}}_{n}\right)$, all axis measurements are rotated to the global coordinate frame $\mathcal{F}_{g}$. Here, axis measurements belonging to the same joint set appear as a cluster. These clusters form an initial estimate of the axis map $\hat{\mathbf{A}}$. 


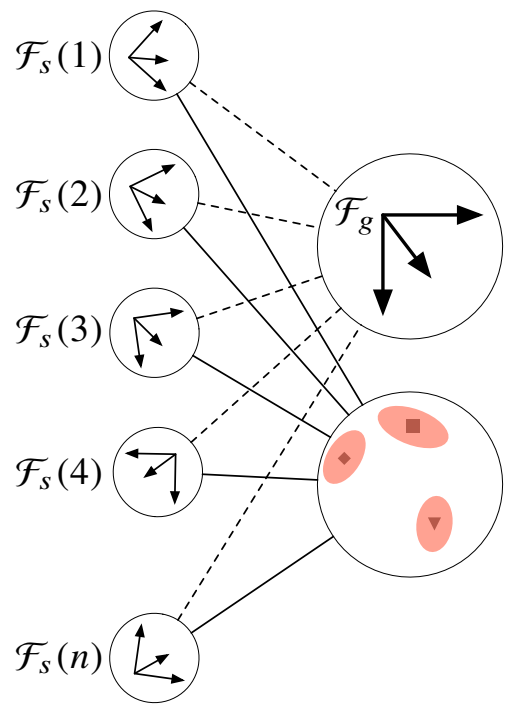

Figure 6: Two different types of measurements are made as the mobile platform traverses the environment. Each dashed line represents an orientation measurement derived from the IMU data (with respect to the global coordinate frame), and each solid line represents an axis measurement derived from the LiDAR data.

\subsection{Building the cost function}

The cost function considers two types of measurements: the orientation measurements (Section 3.3) and axis measurements (Section 3.4). Fig.6 illustrates the two types of measurements over the trajectory of the mobile platform. Like the example in Section 2.2, all the measurements are considered independent and the cost function is the sum of their individual negative log-likelihoods.

The residual error $\mathbf{e}_{k}^{(o)}(\mathbf{x})$ of the $k$-th orientation measurement is

$$
\mathbf{e}_{k}^{(o)}(\mathbf{x})=\tilde{\mathbf{y}}_{k}^{(o)} \boxminus \mathbf{q}_{k},
$$

395

where $\tilde{\mathbf{y}}_{k}^{(o)}$ and $\mathbf{q}_{k}$ are unit quaternions and the $\boxminus$ operator is used in place of subtraction for two purposes: to signify that the difference between two orientations is not their arithmetic difference (in this case, it is a rotation), and to convert the difference to the minimal parameterization. Put differently, the result of (17) is a rotation between the measured and predicted orientation, expressed in a minimal parameterization that behaves like a vector. This parameterization is required to represent the likelihood of the orientation measurement with a Gaussian PDF.

Similarly, the residual error $\mathbf{e}_{j}^{(a)}(\mathbf{x})$ of the $j$-th axis measurement is

$$
\mathbf{e}_{j}^{(a)}(\mathbf{x})=\tilde{\mathbf{y}}_{j}^{(a)} \boxminus \mathbf{C}\left(\mathbf{q}_{k}\right) \mathbf{n}_{j},
$$

where $\tilde{\mathbf{y}}_{j}^{(a)}$ and $\mathbf{n}_{j}$ are unit axes, and $\mathbf{C}\left(\mathbf{q}_{k}\right) \in S O(3)$ is a rotation matrix 
axis measurement, which rotates the axis map entry $\mathbf{n}_{j}$ from $\mathcal{F}_{g}$ to $\mathcal{F}_{s}$. The $\boxminus$ operator is analogous to the one in $(17)$, this time reporting the difference between the measured and predicted axes as an axis vector. Note that only the axis measurements that are associated with an entry of $\mathbf{A}$ contribute to the cost function; the others are considered outliers.

Like the example in Section 2.2, the cost function is the sum of the negative log-likelihood of all the measurements. Using the residual errors in (17) and (18), the cost function is

$$
J(\mathbf{x})=\frac{1}{2} \sum_{k=1}^{n} \mathbf{e}_{k}^{(o)}(\mathbf{x})^{\top} \mathbf{R}_{o}^{-1} \mathbf{e}_{k}^{(o)}(\mathbf{x})+\frac{1}{2} \sum_{j=1}^{p} \mathbf{e}_{j}^{(a)}(\mathbf{x})^{\top} \mathbf{R}_{a}^{-1} \mathbf{e}_{j}^{(a)}(\mathbf{x})
$$

for $n$ orientation measurements and $p$ axis measurements. Because the residual errors are vectors and the measurement uncertainties are represented by covariance matrices, the negative log-likelihood is derived from a multivariate Gaussian distribution. Unfortunately, 19 is not quadratic in the state trajectory and is not easily minimized. Therefore, as is described in Section 3.1 a first order Taylor series expansion is used to linearize the residual errors; i.e.,

$$
\begin{gathered}
\mathbf{e}_{k}^{(o)}(\mathbf{x}) \approx \mathbf{e}_{k}^{(o)}(\hat{\mathbf{x}})+\underbrace{\left.\frac{\partial \mathbf{e}_{k}^{(o)}(\mathbf{x})}{\partial \mathbf{x}}\right|_{\hat{\mathbf{x}}}}_{\mathbf{E}_{k}^{(o)}} \Delta \mathbf{x} \\
\mathbf{e}_{j}^{(a)}(\mathbf{x}) \approx \mathbf{e}_{j}^{(a)}(\hat{\mathbf{x}})+\underbrace{\left.\frac{\partial \mathbf{e}_{j}^{(a)}(\mathbf{x})}{\partial \mathbf{x}}\right|_{\hat{\mathbf{x}}}}_{\mathbf{E}_{j}^{(a)}} \Delta \mathbf{x} .
\end{gathered}
$$

${ }_{420}$ The Jacobians (i.e., the matrices of partial derivatives) $\mathbf{E}_{k}^{(o)}$ and $\mathbf{E}_{j}^{(a)}$ are evaluated numerically. Substituting (20) into the negative log-likelihood of the measurements yields the linearized cost function $J(\hat{\mathbf{x}}, \Delta \mathbf{x})$; i.e.,

$$
J(\hat{\mathbf{x}}, \Delta \mathbf{x})=J_{o}(\hat{\mathbf{x}}, \Delta \mathbf{x})+J_{a}(\hat{\mathbf{x}}, \Delta \mathbf{x}),
$$

where

$$
\begin{aligned}
& J_{o}(\hat{\mathbf{x}}, \Delta \mathbf{x})=\frac{1}{2} \sum_{k=1}^{n}\left(\mathbf{e}_{k}^{(o)}(\hat{\mathbf{x}})+\mathbf{E}_{k}^{(o)} \Delta \mathbf{x}\right)^{\top} \mathbf{R}_{o}^{-1}\left(\mathbf{e}_{k}^{(o)}(\hat{\mathbf{x}})+\mathbf{E}_{k}^{(o)} \Delta \mathbf{x}\right) \\
& J_{a}(\hat{\mathbf{x}}, \Delta \mathbf{x})=\frac{1}{2} \sum_{j=1}^{p}\left(\mathbf{e}_{j}^{(a)}(\hat{\mathbf{x}})+\mathbf{E}_{j}^{(a)} \Delta \mathbf{x}\right)^{\top} \mathbf{R}_{a}^{-1}\left(\mathbf{e}_{j}^{(a)}(\hat{\mathbf{x}})+\mathbf{E}_{j}^{(a)} \Delta \mathbf{x}\right),
\end{aligned}
$$

which is quadratic with respect to the state trajectory perturbation $\Delta \mathbf{x}$.

\subsection{Minimizing the cost function}

Minimizing the cost function is an iterative process. Because the linearized cost function is quadratic with with respect to $\Delta \mathbf{x}$, it is relatively straightforward 
to solve for the optimal state trajectory perturbation $\Delta \mathrm{x}^{*}$. However, because $\Delta \mathrm{x}^{*}$ only minimizes the linearized cost function in (21) and not the actual cost function in (19), it can not be guaranteed that perturbing $\hat{\mathbf{x}}$ by $\Delta \mathrm{x}^{*}$ will actually yield an improvement. As a result, one must alternate between solving for $\Delta \mathbf{x}^{*}$, and checking whether applying the perturbation reduces the cost of the actual cost function. Several algorithms exist that perform these iterations using various heuristics to reach a solution faster or more robustly. 3DAM uses 435 a variant of the Levenberg-Marquardt algorithm [29] for this purpose.

Recall that the state trajectory $\mathbf{x}$ contains the orientations of the mobile platform and the axes of the joint sets. Therefore, when a perturbation $\Delta \mathrm{x}^{*}$ is applied to the state trajectory to update its estimate, the outcome of several of the steps in the previous sections that used the state trajectory estimate will change. For example, Fig. Fillustrates how the axis measurements are rotated to the global frame using $\mathbf{Q}$. Now that $\mathbf{Q}$ has changed, this step must be redone. In fact, all steps involving $\hat{\mathbf{x}}=(\hat{\mathbf{Q}}, \hat{\mathbf{A}})$ are redone using the perturbed state trajectory estimate, and this process is repeated until convergence (e.g., $\Delta \mathbf{x}^{*}$ is negligibly small). The resulting state trajectory estimate $\hat{\mathbf{x}}^{*}$ contains the most

445 likely orientations of the mobile platform and the most likely axes of the joint sets.

\subsection{Generating the stereonet}

Recall that an axis measurement is a cluster of similar point axes extracted from a LiDAR measurement (Fig.44. In Section 3.4. each LiDAR measurement 450 is reduced to a small number of axis measurements that is representative of the surfaces that were measured. For example, if the LiDAR were to scan a flat wall, it would produce thousands of similar point axes that would be clustered into a single axis observation. Likewise, a scan of a rock face produces thousands of point axes that form clusters primarily at the joint sets, becauseby definition - the joint sets manifest themselves as sets of similarly oriented planar surfaces. As a result, each LiDAR measurement is a snapshot of the joint sets that were visible in the field of view of the sensor. A stereonet is generated by putting all of these snapshots together.

The optimal estimate of the orientations $\hat{\mathbf{Q}}^{*}$ calculated in Section 3.6 is a list containing the orientation of the LiDAR at each snapshot, where each snapshot contains a small number of axis measurements. Each entry of $\hat{\mathbf{Q}}^{*}$ is used to rotate the axis measurements in its respective snapshot to the global coordinate frame. Next, each axis measurement is converted to dip and dip direction using (3) and is plotted on a stereonet. This process is illustrated in Fig.7.

\section{Field trials}

Field trials were performed at three different field sites, two of which employed a handheld configuration of the sensors and one which employed a remotely operated mobile robot. This section provides a detailed description of both the apparatus and the field sites. 


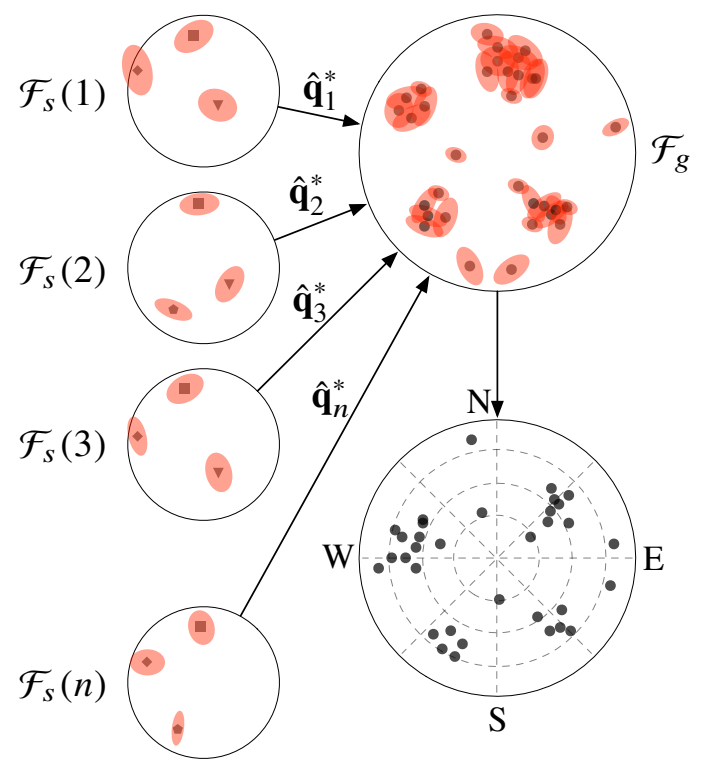

Figure 7: The conversion of axis measurements into a stereonet. The optimal estimate of the orientations $\hat{\mathbf{Q}}^{*}=\left(\hat{\mathbf{q}}_{1}^{*}, \ldots \hat{\mathbf{q}}_{n}^{*}\right)$ of the mobile platform coordinate frame $\mathcal{F}_{s}$ at each LiDAR measurement are used to rotate all axis measurements to the global coordinate frame $\mathcal{F}_{g}$. The relationships in (3) are then used to convert the axis measurements to dip and dip direction, at which point they are plotted on a stereonet. 


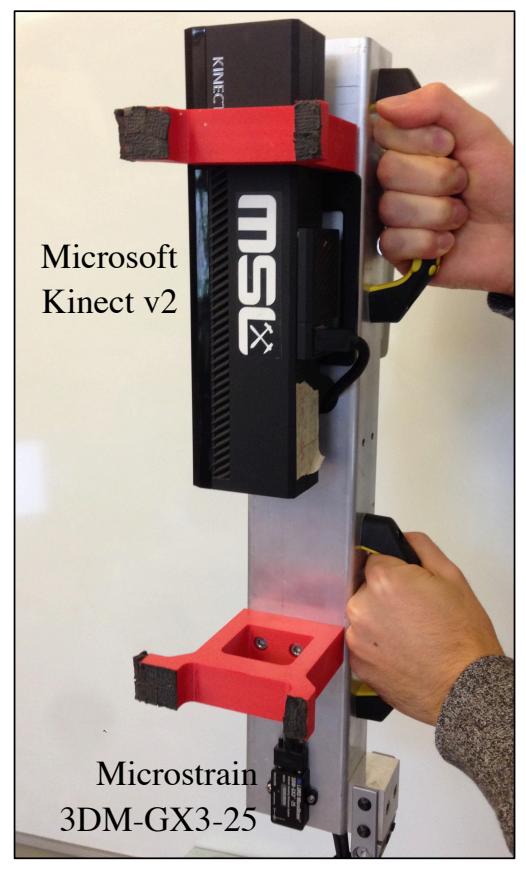

Figure 8: The 3DAM mobile platform used for the experiments. A Microsoft Kinect v2 time-of-flight camera (scannerless LiDAR) and a LORD MicroStrain 3DM-GX3-25 IMU are mounted to a piece of aluminum extrusion. The attached handles allow for handheld operation.

\subsection{Apparatus}

A mobile platform was constructed consisting of a Microsoft Kinect v2 timeof-flight camera [30] and a LORD MicroStrain 3DM-GX3-25 IMU [31]. The two sensors were rigidly connected on a short length of aluminum extrusion, which was outfitted with handles for handheld use (Fig.8). The sensors were wired to a laptop and a small battery carried inside a backpack, and a tablet computer was connected to the mobile platform that outputted information to user (sensor status, distance from rock face, estimated planarity of point cloud, and acceleration). This version of the mobile platform was a proof-of-concept prototype, and it is conceivable that it could be made much more compact.

Time-of-flight cameras are a form of scannerless LiDAR, which acquire data from the full field of view simultaneously as opposed to capturing the point cloud one point of a time. The use of scannerless LiDAR is what allows the mobile platform to be in motion during data collection. Specifications of the Microsoft Kinect v2 are listed in Table 1. Note that the noise depends on both the range and operating conditions. An in-depth analysis of the noise characteristics of was performed by Fankhauser et al. 32. Comprehensive specifications of the LORD MicroStrain 3DM-GX3-25 IMU are available in its manual [33].

In one experiment, a stereonet was generated by processing the point cloud measured by an MDL (now Renishaw) Quarryman Pro 3D laser scanner 34] 
Table 1: Specifications of the Microsoft Kinect v2.

\begin{tabular}{lr}
\hline Specification & Value \\
\hline Dimensions (mm) & $249 \times 66 \times 67$ \\
Mass (kg) & 0.970 \\
Resolution (pixels) & $512 \times 424$ \\
Min. range (m) & 0.5 \\
Max. range (m) & 4.5 \\
Horiz. field of view (deg) & 70 \\
Vert. field of view (deg) & 60 \\
Axial and lateral noise (mm) & $<10$ \\
Max. frequency (frames per second) & 30 \\
Power usage (W) & 15 \\
Cost (USD) & 200 \\
\hline
\end{tabular}

(stationary time-of-flight LiDAR). More details about this process are described in Section 4.2 and its results are presented in Section 5.2 .

\subsection{Experiments}

Three experiments were performed to test the facets of 3DAM and to compare its results to other methods of joint orientation estimation. The locations of these experiments were

(i) a road cut near Brewer Lake $\left(44^{\circ} 24^{\prime} 04.4^{\prime \prime} \mathrm{N}, 76^{\circ} 18^{\prime} 46.9^{\prime \prime} \mathrm{W}\right)$, approximately $25 \mathrm{~km}$ northeast of Kingston, ON, Canada;

(ii) a road cut near Ivy Lea $\left(44^{\circ} 22^{\prime} 13.2^{\prime \prime} \mathrm{N}, 75^{\circ} 58^{\prime} 37.5^{\prime \prime} \mathrm{W}\right)$, approximately $45 \mathrm{~km}$ east of Kingston, ON, Canada;

(iii) an old railway tunnel on the Kettle Valley Trail in Myra-Bellevue Provincial Park $\left(49^{\circ} 46^{\prime} 58.5^{\prime \prime} \mathrm{N}, 119^{\circ} 18^{\prime} 29.2^{\prime \prime} \mathrm{W}\right)$, approximately $20 \mathrm{~km}$ southeast of Kelowna, BC, Canada.

The $19.5 \mathrm{~m} \times 2.5 \mathrm{~m}$ section of the road cut at Brewer Lake used in the experiments is pictured in Fig.9. Brewer lake was selected as a test site because joint orientation estimation was previously performed on the same rock face by Lato et al. [5], which included results from both hand measurements (compass and inclinometer) and stationary LiDAR. These results are compared with 3DAM in Section 5.1 .

The $6.1 \mathrm{~m} \times 2.5 \mathrm{~m}$ section of the road cut at Ivy Lea used in the experiments is pictured in Fig.10. In addition to hand measurements and the data collected for 3DAM, a point cloud of the rock face was collected using the MDL Quarryman Pro. A stereonet was generated by extracting the point axes of this point cloud using the same algorithm that is used in 3DAM (as described in Section 3.4 and illustrated in Fig.44. Results of these different methods of joint orientation estimation are presented in Section 5.2 . 


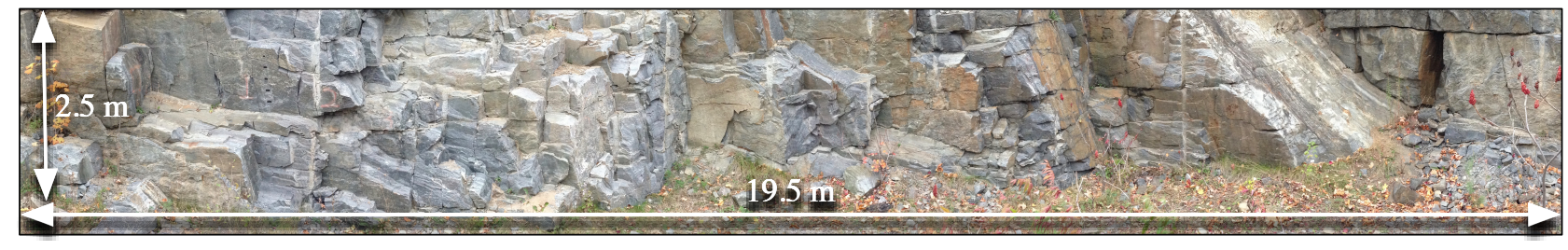

Figure 9: The section of rock face at Brewer Lake used in the joint orientation estimation experiments.

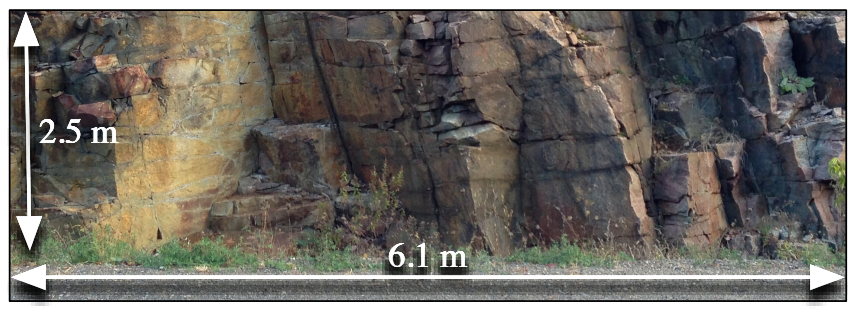

Figure 10: The section of rock face at Ivy Lea used in the joint orientation estimation experiments. Note that a small ditch occludes the bottom portion of the rock face in this picture.

The 3DAM hardware shown in Fig.8 8 was mounted on a Clearpath Husky A200 mobile robot and driven through an old railway tunnel in Myra-Bellevue (pictured in Fig.11). The robot was remotely driven along a $6 \mathrm{~m}$ section of the tunnel wall at approximately $0.5 \mathrm{~m} / \mathrm{s}$ using a joystick. Hand measurements were taken at the same section of the tunnel and the results are presented in Section 5.3

At Brewer Lake and Ivy Lea, data was collected with the 3DAM mobile platform in two different ways: biased and unbiased handheld LiDAR scanning. In the biased experiments, the operator smoothly moved the mobile platform ${ }_{525}$ through the environment while deliberately pointing the LiDAR at planar surfaces. In the unbiased experiments, the operator moved the mobile platform in the predefined pattern illustrated in Fig.12. In the Myra-Bellevue experiments, the mobile robot was simply driven in a straight line roughly parallel to the tunnel wall. In all experiments, data was collected with the LiDAR at two frames per second and with the IMU at $100 \mathrm{~Hz}$.

\section{Results and discussion}

This section presents the results of the individual experiments at Brewer Lake (Section 5.1), Ivy Lea (Section 5.2), and Myra-Bellevue (Section 5.3). Multiple trials of 3DAM were performed at each location but, for brevity's sake, the results of only a single typical trial are reported. The dips and dip directions of the joint sets presented in this section were calculated using the fuzzy cluster analysis tool in the Rocscience Dips software package [35. This 


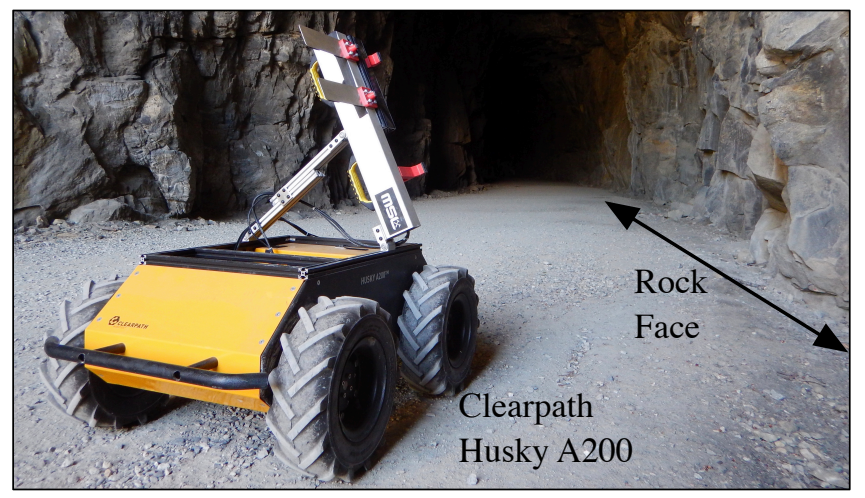

Figure 11: The Clearpath Husky A200 mobile robot in the old railway tunnel in MyraBellevue. The 3DAM mobile platform illustrated in Fig.8 is mounted on top of the robot. The rock face used in the joint orientation estimation experiments is shown the background.

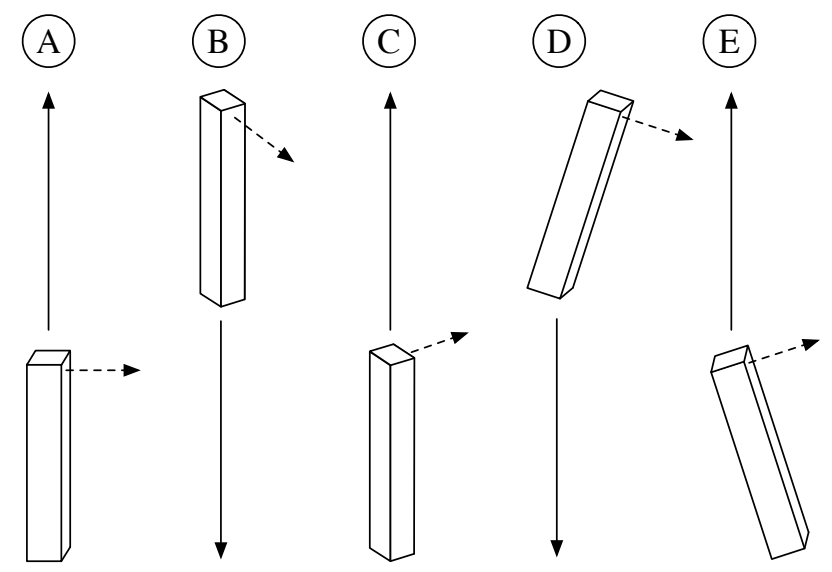

Figure 12: The scanning pattern used in the unbiased 3DAM experiments. The dashed arrows indicate the direction of measurement and the solid arrows indicate the direction of movement of the mobile platform. The pattern consists of the operator moving the LiDAR as follows: (A) face the rock face and raise; (B) rotate to approximately $30^{\circ}$ right of centre and lower; (C) rotate to approximately $30^{\circ}$ left of centre and raise; (D) rotate to approximately $30^{\circ}$ down of centre and lower; (E) rotate to approximately $30^{\circ}$ up of centre and raise. The operator then steps approximately $1-2 \mathrm{~m}$ to the side and repeats the pattern. This process continues until the full rock face has been scanned. 
Table 2: Results of the Brewer Lake experiments.

\begin{tabular}{lrrrr}
\hline & $\begin{array}{r}\text { Hand } \\
\text { measurements }\end{array}$ & $\begin{array}{r}\text { Stationary } \\
\text { LiDAR }\end{array}$ & $\begin{array}{r}\text { Biased } \\
\text { 3DAM }\end{array}$ & $\begin{array}{r}\text { Unbiased } \\
\text { 3DAM }\end{array}$ \\
\hline Number of poles & 101 & 257 & 605 & 688 \\
& & & & \\
Joint orientations & & & & \\
(dip / dip direction) & $77 / 137$ & $61 / 134$ & $77 / 134$ & $75 / 137$ \\
Set 1 & $89 / 060$ & $83 / 056$ & $89 / 234$ & $89 / 057$ \\
Set 2 & $28 / 314$ & $39 / 308$ & $40 / 302$ & $22 / 297$ \\
Set 3 & & & & \\
& & & & \\
Angular difference with respect & - & 16.2 & 2.9 & 2.0 \\
to hand measurements (deg) & - & 7.2 & 6.3 & 3.0 \\
Set 1 & - & 11.5 & 13.7 & 9.3 \\
Set 2 & & & & \\
Set 3 & 0 & $\sim 30^{\mathrm{a}}$ & $5^{\mathrm{b}}$ & $5^{\mathrm{b}}$ \\
& $\sim 120^{\mathrm{c}}$ & $\sim 105^{\mathrm{a}}$ & 1 & 3 \\
Approximate effort (min) & 10 & $\sim 180^{\mathrm{a}}$ & 10 & 10 \\
Setup / take down & $\sim 130$ & $\sim 315$ & 16 & 18 \\
$\quad$ Data collection & & & \\
$\quad$ Data processing & & & & \\
Total & & & & \\
\hline
\end{tabular}

a These times are not reported in [5]. Times are estimated based on those reported in a similar experiment using the top down approach [10].

b Setup only needs to be performed once for multiple trials.

${ }^{c}$ This time is not reported in [5. This number is estimated based on the experience of the authors and the number of measurements.

tool automatically determines the poles belonging to a joint set within a 30

degree radius of a highly concentrated area in a stereonet and reports the mean

dip and dip direction of the resulting cluster.

\subsection{Brewer Lake}

The results of four different joint orientation estimation experiments are compared and discussed in this section:(i) hand measurements using a compass and inclinometer (Fig.13a); (ii) the top-down approach using a stationary Li-

545 DAR (Fig.13b); (iii) biased 3DAM (Fig.13c); (iv) unbiased 3DAM (Fig.13d). The results of (i) and (ii) are reproduced from [5] with the permission of the authors. Note that the reproduced stereonets differ slightly compared to those in [5] because the originals did not account for the local magnetic declination. A summary of the results is provided in Table 2.

550 The orientations of the three joint sets extracted from both the biased and unbiased 3DAM stereonets are comparable to those extracted with hand mea- 


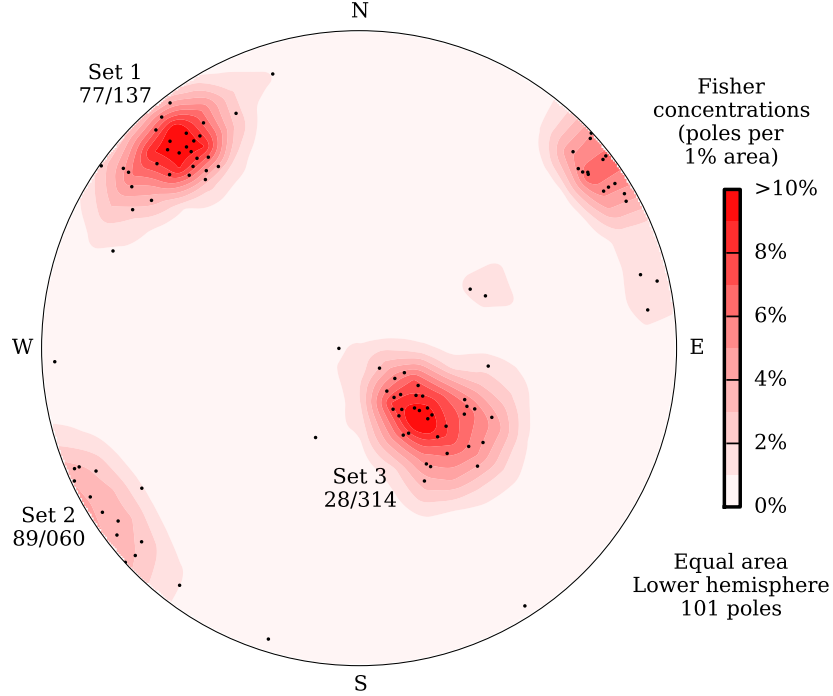

(a) Hand measurements using a compass and inclinometer.

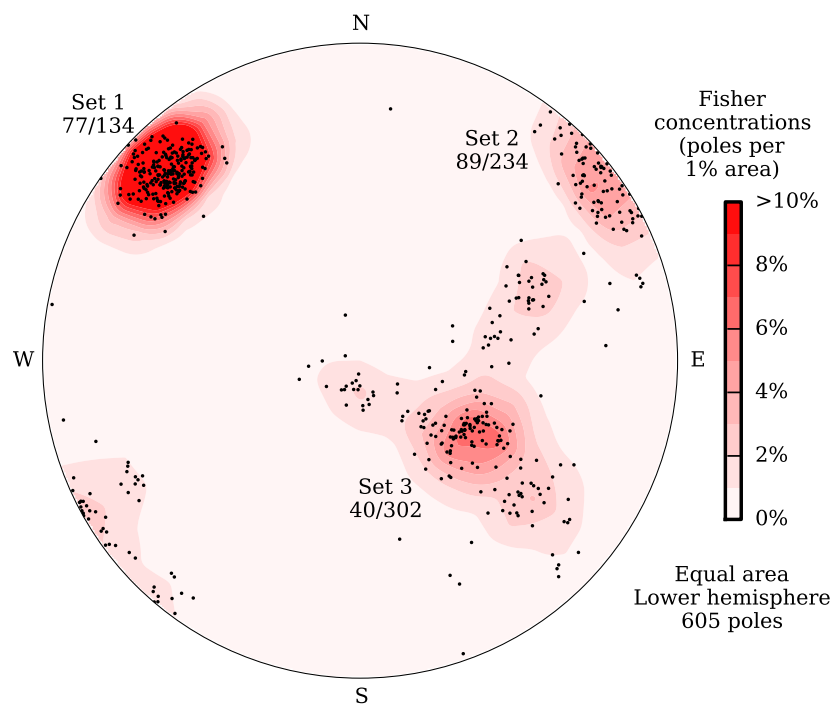

(c) Biased 3DAM.

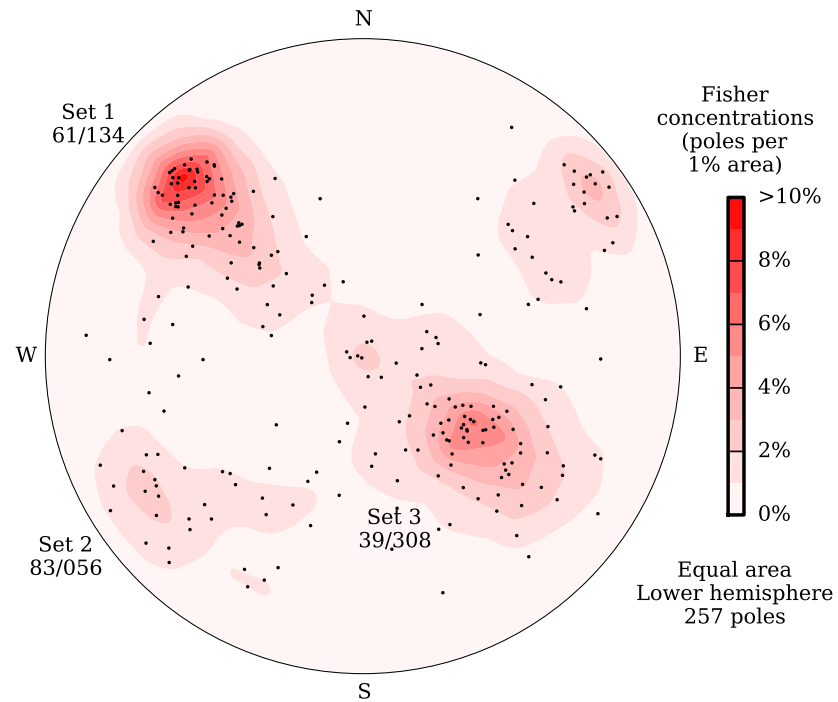

(b) Top-down approach using a stationary LiDAR.

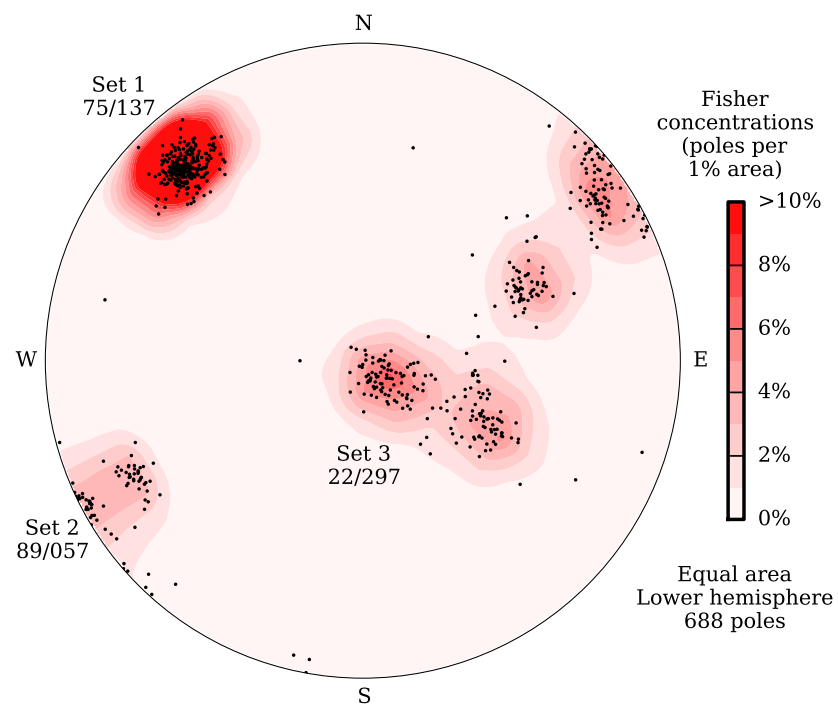

(d) Unbiased 3DAM.

Figure 13: Equal area, lower hemisphere stereonets illustrating the four different joint orientation estimation methods used in the Brewer Lake experiments. 
surements. The largest deviation between 3DAM and the hand measurements is the orientation of Set 3. A major contributing factor to this discrepancy is the continuum of poles between Sets 2 and 3 in the 3DAM results, which perturbs the dip direction of Set 3 and is not present in the hand measurements. It should be noted that these poles are present in all trials of both the biased and unbiased 3DAM. The presence of only two poles between Sets 2 and 3 in the hand measurements suggest planar surfaces at this orientation were possibly undersampled by the hand measurements.

Compared to the results of the top-down approach, the 3DAM stereonets have considerably less scatter and a greater concentration of poles at the three joint sets. This suggests that the robust bottom-up approach used by 3DAM is effective at rejecting points measuring non-planar surfaces, which parallels the conclusions of Slob [15. Additionally, the similarity of the biased and unbiased 3DAM results implies that deliberately pointing the LiDAR at planar surfaces is not necessarily required. This suggests that $3 \mathrm{DAM}$ is effective at ignoring the additional non-planar surfaces that may be measured by an unbiased scanning pattern, which again is likely a consequence of the bottom-up extraction of joint orientations.

Perhaps the most significant difference between 3DAM and other methods of joint orientation estimation is the amount of effort required. Stationary LiDAR often requires multiple scans from different viewpoints in order to avoid occlusions, which can introduce bias to the extracted joint sets [7. Fig. $13 \mathrm{~b}$ is the result of combining seven separate LiDAR measurements from three different 575 locations. Each scan adds effort both in the field and when processing the data. Conversely, 3DAM requires substantially less effort; in fact, the actual data collection took only a few minutes in each experiment. This is the result of using scannerless LiDAR (capturing a point cloud takes a fraction of a second instead of several minutes) and the mobility of 3DAM (occlusions are eliminated by simply and easily maneuvering the device).

\subsection{Ivy Lea}

This section compares and discusses the results of four different joint orientation estimation experiments:(i) hand measurements using a compass and inclinometer (Fig.14a); (ii) the 3DAM bottom-up approach using a stationary

${ }_{585}$ LiDAR (Fig.14b); (iii) biased 3DAM (Fig.14c); (iv) unbiased 3DAM (Fig.14d). The joint orientations determined in (ii) are the point axes extracted from a single scan of the stationary LiDAR, using the same bottom-up approach employed in (iii) and (iv). A summary of the results is provided in Table 3.

All three LiDAR based methods produced joint set orientations similar to those resulting from the manual measurements. In fact, for Sets 1 and 2, the angular differences are less than five degrees. However, the angular differences relative to Set 3 are more significant. It is speculated that a major contribution to this discrepancy is the difficulty of accurately measuring the dip direction by hand on surfaces with small dips. On these surfaces, the measured dip direction 
Table 3: Results of the Ivy Lea experiments.

\begin{tabular}{lrrrr}
\hline & $\begin{array}{r}\text { Hand } \\
\text { measurements }\end{array}$ & $\begin{array}{r}\text { Stationary } \\
\text { LiDAR }\end{array}$ & $\begin{array}{r}\text { Biased } \\
\text { 3DAM }\end{array}$ & $\begin{array}{r}\text { Unbiased } \\
\text { 3DAM }\end{array}$ \\
\hline Number of poles & 70 & 18101 & 359 & 1229 \\
& & & & \\
Joint orientations & & & & \\
(dip / dip direction) & $90 / 352$ & $86 / 354$ & $88 / 169$ & $89 / 171$ \\
Set 1 & $73 / 069$ & $72 / 066$ & $75 / 065$ & $76 / 066$ \\
Set 2 & $11 / 203$ & $23 / 237$ & $14 / 253$ & $17 / 234$ \\
Set 3 & & & & \\
& & & & \\
Angular difference with respect & - & 4.5 & 3.6 & 1.4 \\
to hand measurements (deg) & - & 3.0 & 4.3 & 4.2 \\
Set 1 & - & 15.1 & 10.8 & 9.4 \\
Set 2 & & & & \\
Set 3 & & & & \\
& & & 5 & \\
Approximate effort (min) & $\sim 85$ & $40^{\mathrm{b}}$ & 2 & 4 \\
Setup / take down & 10 & 10 & 10 & 10 \\
Data collection & 95 & 60 & 17 & 19 \\
Data processing & & & & \\
Total & & & & \\
\hline
\end{tabular}

${ }^{a}$ Setup only needs to be performed once for multiple trials.

$\mathrm{b}$ This time depends on the desired resolution of the resulting point cloud. In this case, the point cloud was downsampled so data collection could have been performed faster.

and accessibility of the point of contact. Note that the offset of the dip direction in all three LiDAR based methods is in the same direction.

It appears that that the stationary LiDAR was well-oriented to capture all three joint sets. Due to the local terrain, the LiDAR stood slightly above 600 the centre of the scanned area, allowing for a better line of sight of the nearlyhorizontal Set 3. Note that no manual pruning of the point cloud was performed and points belonging to planar surfaces are discovered automatically.

\subsection{Myra-Bellevue}

The purpose of the Myra-Bellevue experiment is to demonstrate that 3DAM can be performed by remotely operating a vehicle to collect the data. In this section, joint orientation estimation using this approach (Fig. 15b) is compared with hand measurements using a compass and inclinometer (Fig.15a). A summary of the results is provided in Table 4.

Compared to the results at Brewer Lake and Ivy Lea, the angular differences ${ }_{610}$ between the hand measured and 3DAM joint sets are slightly larger at MyraBellevue. One possible cause of this discrepancy is the path driven by the robot. 


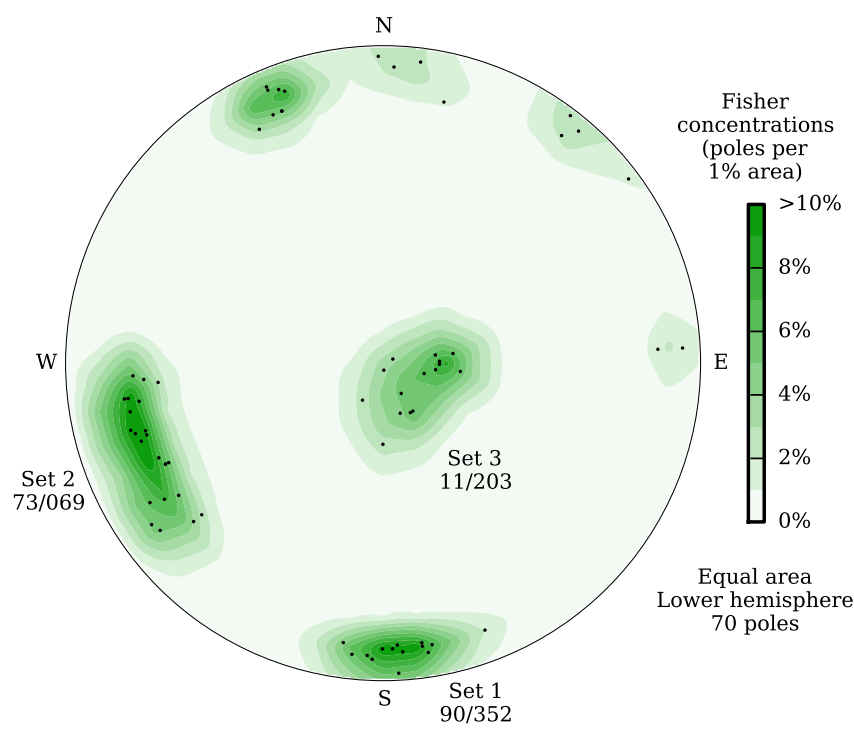

(a) Hand measurements using a compass and inclinometer.

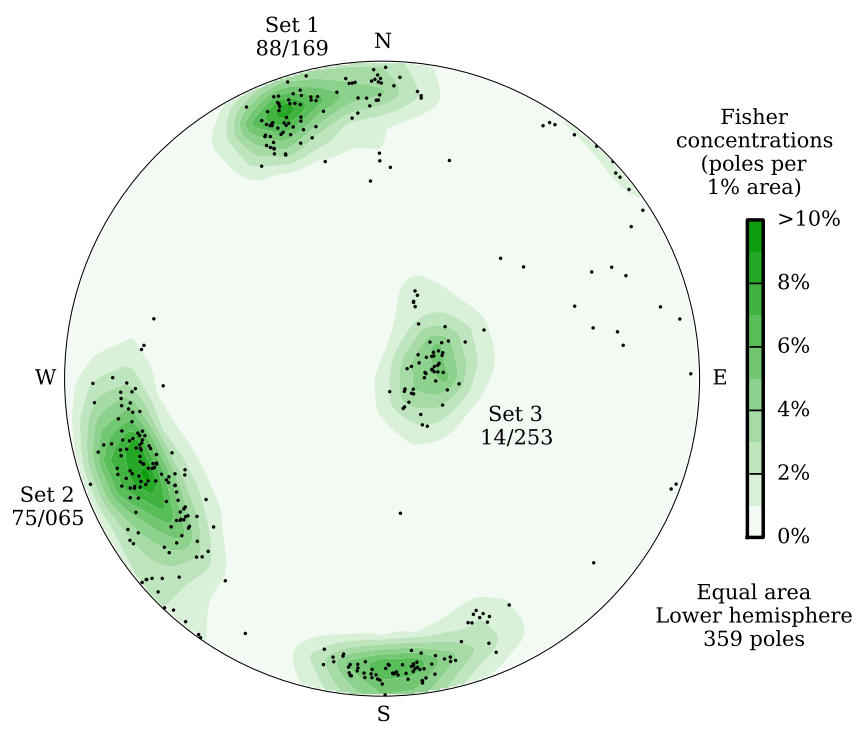

(c) Biased 3DAM.

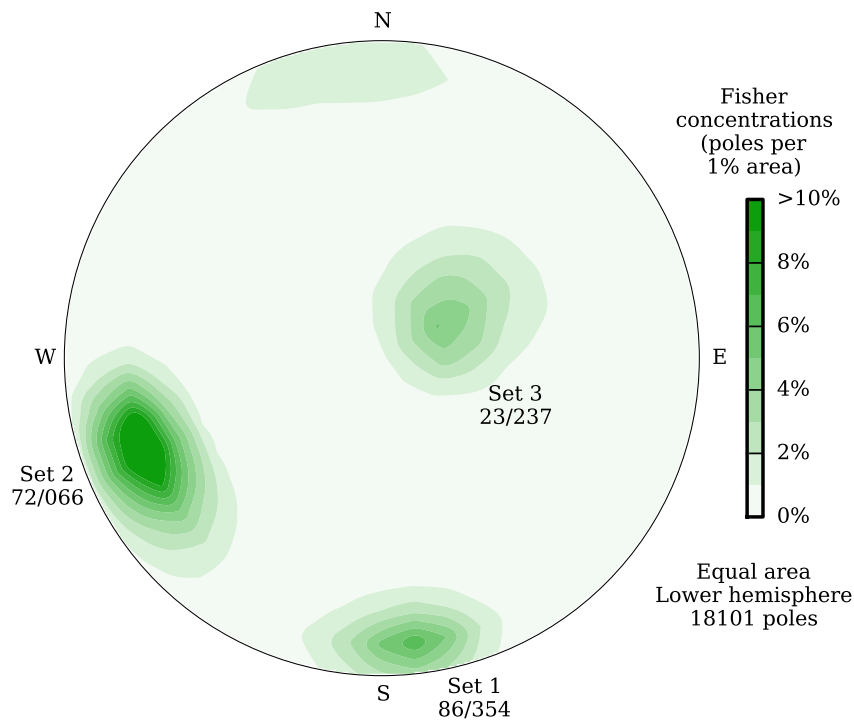

(b) Point axes extracted from stationary LiDAR point cloud.

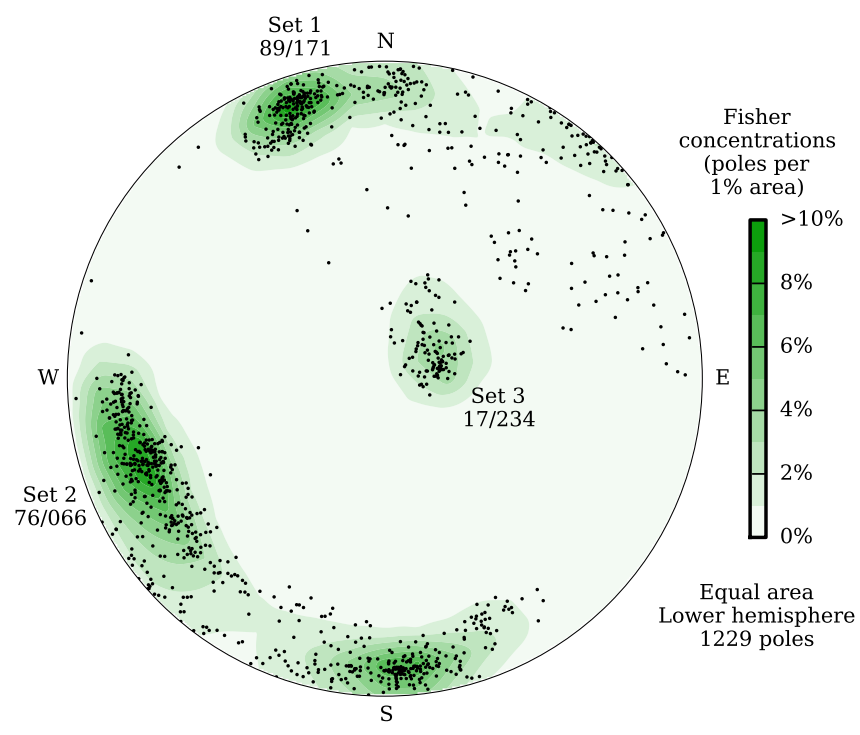

(d) Unbiased 3DAM.

Figure 14: Equal area, lower hemisphere stereonets illustrating the four different joint orientation estimation methods used in the Ivy Lea experiments. 


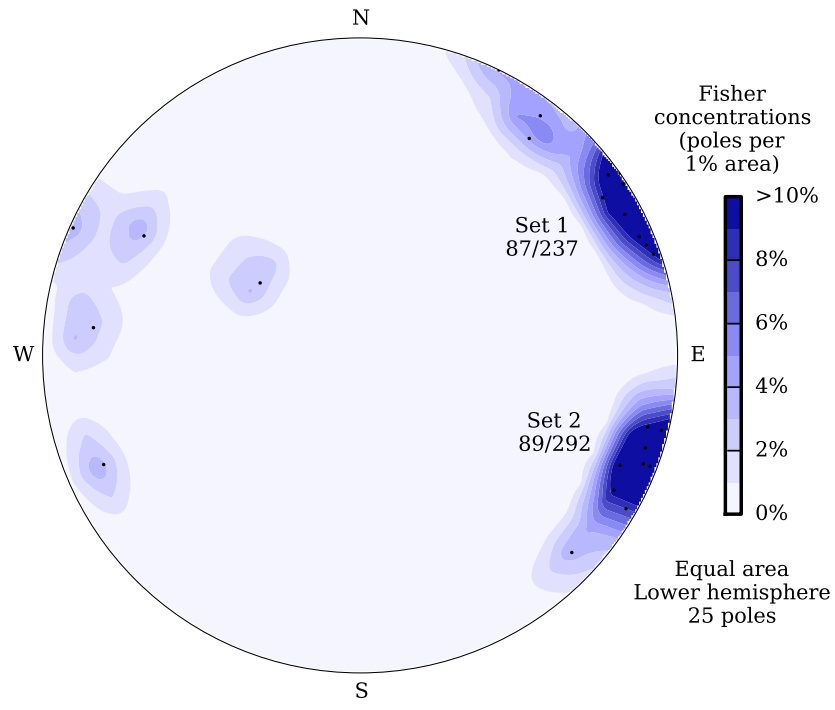

(a) Hand measurements using a compass and inclinometer.

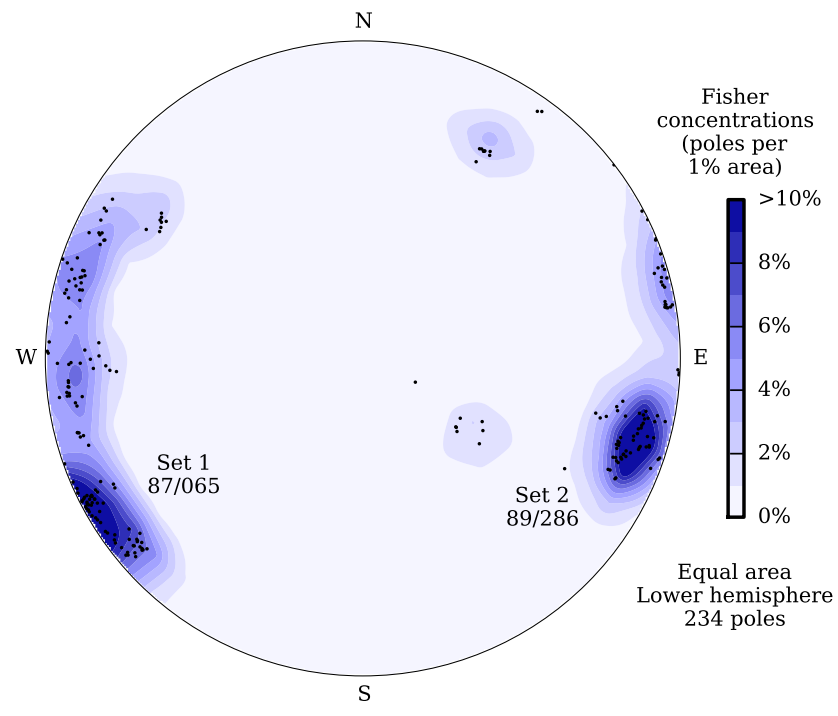

(b) 3DAM using a mobile robot.

Figure 15: Equal area, lower hemisphere stereonets illustrating the two different joint orientation estimation methods used in the Myra-Bellevue experiments. 
Table 4: Results of the Myra-Bellevue experiments.

\begin{tabular}{lrr}
\hline & $\begin{array}{r}\text { Hand } \\
\text { measurements }\end{array}$ & $\begin{array}{r}\text { 3DAM using a } \\
\text { mobile robot }\end{array}$ \\
\hline Number of poles & 25 & 234 \\
Joint orientations & & \\
(dip / dip direction) & & \\
Set 1 & $87 / 237$ & $87 / 065$ \\
Set 2 & $89 / 292$ & $89 / 286$ \\
& & \\
Angular difference with respect & & \\
to hand measurements (deg) & - & \\
Set 1 & - & \\
Set 2 & & $10^{\mathrm{a}}$ \\
& & 1 \\
Approximate effort (min) & 0 & 10 \\
Setup / take down & 45 & 21 \\
Data collection & 10 & \\
Data processing & 55 & \\
Total & & \\
\hline
\end{tabular}

a Setup only needs to be performed once for multiple trials. 
Unlike the biased and unbiased 3DAM used in the previous experiments, the orientation of the LiDAR was relatively constant while the robot was driven in a straight line roughly parallel to the rock face. In particular, as pictured in ${ }_{615}$ Fig.11, the dip of the LiDAR was fixed. It is speculated that performing 3DAM from a remotely operated robot could be improved by ensuring there is a greater variety of viewpoints of the rock face. This could be achieved by actuating the LiDAR, deliberately maneuvering the robot relative to the scanned surfaces, or using a robot with more degrees of freedom in its motion (e.g., a flying robot).

\section{Conclusion}

This paper demonstrates how to automatically and rapidly map joint orientations with a mobile LiDAR by using a new technique called three-dimensional axis mapping (3DAM). The hardware necessary for 3DAM is widely available, low-cost when compared with traditional tripod-mounted (i.e., stationary) scan-

625 ners, and even small enough for a human to easily carry and maneuver. What is more, the described system is deployable on a mobile robot in order to access areas that may be dangerous or beyond the reach of human operators. The main tradeoff is the comparatively short range $(<4.5 \mathrm{~m})$ of the employed time-of-flight camera technology.

As described in detail, the 3DAM algorithm works by solving a global state estimation problem that simultaneously finds an optimal estimate the orientations of the mobile device together with measured joint set orientations. Extensive field experiments were conducted in three different environments, and this paper compares the final 3DAM output with hand (compass and inclinome-

${ }_{635}$ ter) measurements, stationary tripod-mounted LiDAR, as well as with recently published results on LiDAR-based stereonet generation by other researchers. In all instances, 3DAM clearly outperforms other methods not only in the quality of the output, but also in the extremely short time needed to generate a useful stereonet. Although the hardware configuration used in the experiments ${ }^{4} 40$ described by this paper was a utilitarian prototype, it is foreseeable that a commercial version could be developed based on a small tablet computer or smartphone.

\section{Acknowledgements}

The authors would like to acknowledge Heshan Fernando for helping with hardware design, Glen Turner for helping with field data collection in Kelowna, and James Procopio for helping with LiDAR data collection in Kingston using the MDL Quarryman Pro. Thanks to Matthew Lato for permission to use his data from Brewer Lake, and to Steve McKinnon for many useful conversations about joint sets and stereonets.

This research was funded in part by the Natural Sciences and Engineering Research Council of Canada (NSERC) under grant 397985-2011 and the NSERC Canadian Field Robotics Network (NCFRN) project NETGP 417354-11. 


\section{References}

[1] J. Kemeny, K. Turner, Ground-based LiDAR rock slope mapping and assessment, Tech. Rep. FHWA-CFL/TD-08-006, U.S. Department of Transportation Federal Highway Administration, Lakewood, CO (Sep. 2008).

[2] R. Roncella, G. Forlani, Extraction of planar patches from points clouds to retrieve dip and dip direction of rock discontinuities, in: ISPRS WG III/3, III/4, V/3 Workshop "Laser scanning 2005", Enschede, Netherlands, 2005, pp. $162-167$.

[3] M. I. Olariu, J. F. Ferguson, C. L. V. Aiken, X. Xu, Outcrop fracture characterization using terrestrial laser scanners: Deep-water Jackfork sandstone at Big Rock Quarry, Arkansas, Geosphere 4 (1) (2008) 247-259.

[4] A. M. Ferrero, G. Forlani, R. Roncella, H. I. Voyat, Advanced geostructural survey methods applied to rock mass characterization, Rock Mechanics and Rock Engineering 42 (2) (2009) 631-665.

[5] M. Lato, M. S. Diederichs, D. J. Hutchinson, R. Harrap, Optimization of LiDAR scanning and processing for automated structural evaluation of discontinuities in rockmasses, International Journal of Rock Mechanics \& Mining Sciences 46 (1) (2009) 194-199.

[6] M. Sturzenegger, D. Stead, Quantifying discontinuity orientation and persistence on high mountain rock slopes and large landslides using terrestrial remote sensing techniques, Nat. Hazards Earth Syst. Sci. 9 (2) (2009) 267287.

[7] M. Lato, M. S. Diederichs, D. J. Hutchinson, Bias correction for viewlimited Lidar scanning of rock outcrops for structural characterization, Rock Mechanics and Rock Engineering 43 (5) (2010) 615-628.

[8] D. García-Sellés, O. Falivene, P. Arbués, O. Gratacos, S. Tavani, J. A. Muñoz, Supervised identification and reconstruction of near-planar geological surfaces from terrestrial laser scanning, Computers \& Geosciences 37 (10) (2011) 1584-1594.

[9] G. Gigli, N. Casagli, Semi-automatic extraction of rock mass structural data from high resolution LIDAR point clouds, International Journal of Rock Mechanics \& Mining Sciences 48 (2) (2011) 187-198.

[10] J. Mah, C. Samson, S. D. McKinnon, 3D laser imaging for joint orientation analysis, International Journal of Rock Mechanics \& Mining Sciences 48 (6) (2011) 932-941.

[11] S. García-Cortés, C. Ordóñez Galán, R. Argüelles-Fraga, A. Menéndez Díaz, Automatic detection of discontinuities from 3D point clouds for the stability analysis of jointed rock masses, in: Proceedings of the 18th International Conference on Virtual Systems and Multimedia, IEEE, Milan, Italy, 2012, pp. 595-598. 
[12] M. J. Lato, M. Vöge, Automated mapping of rock discontinuities in 3D lidar and photogrammetry models, International Journal of Rock Mechanics \& Mining Sciences 54 (2012) 150-158.

[13] InnovMetric Software, Polyworks, http://www.innovmetric.com (201510-05).

[14] S. Slob, B. van Knapen, R. Hack, K. Turner, An approach to automate discontinuity measurements of rock faces using lasers scanning techniques, in: Eurock 2002 ISRM International Symposium on Rock Engineering for Mountainous Regions, Funchal, Portugal, 2002, pp. 87-94.

[15] S. Slob, Automated rock mass characterisation using 3-D terrestrial laser scanning, Ph.D. thesis, Delft University of Technology, Delft, Netherlands (Jun. 2010).

[16] M. De Agostino, A. Lingua, M. Piras, Rock face surveys using a LiDAR MMS, Italian Journal of Remote Sensing 44 (1) (2012) 141-151.

[17] C. Glennie, Reign of point clouds, InsideGNSS (2007) 22-31.

[18] M. Lato, J. Hutchinson, M. Diederichs, D. Ball, R. Harrap, Engineering monitoring of rockfall hazards along transportation corridors: using mobile terrestrial LiDAR, Nat. Hazards Earth Syst. Sci. 9 (3) (2009) 935-946.

[19] J. L. Crassidis, J. L. Junkins, Optimal Estimation of Dynamic Systems, 2nd Edition, CRC Press, Boca Raton, FL, 2012.

[20] J. B. Kuipers, Quaternions and Rotation Sequences, Princeton University Press, Princeton, NJ, 1999.

[21] F. L. Markley, Attitude error representations for Kalman filtering, Journal of Guidance, Control, and Dynamics 26 (2) (2003) 311-317.

[22] H. Durrant-Whyte, T. Bailey, Simultaneous localization and mapping: Part I, IEEE Robotics \& Automation Magazine 13 (2) (2006) 99-110.

[23] J. Stewart, Calculus, 7th Edition, Brooks/Cole, Belmont, CA, 2012.

[24] N. Trawny, S. I. Roumeliotis, Indirect Kalman filter for 3D attitude estimation, Tech. Rep. 2005-002, Rev. 57, MARS Lab, Department of Computer Science and Engineering, University of Minnesota, Minneapolis, MN (Mar. 2005).

[25] A. Nurunnabi, G. West, D. Belton, Outlier detection and robust normalcurvature estimation in mobile laser scanning 3D point cloud data, Pattern Recognition 48 (2014) 1404-1419.

[26] I. Jolliffe, Principal Component Analysis, 2nd Edition, Springer Series in Statistics, Springer-Verlag, New York, NY, 2012. 
[27] M. Ester, H.-P. Kriegel, J. Sander, X. Xu, A density-based algorithm for discovering clusters in large spatial databases with noise, in: Proceedings of the 2nd International Conference on Knowledge Discovery and Data Mining, AAAI Press, Portland, OR, 1996, pp. 226-231.

[28] R. Beran, N. I. Fisher, Nonparametric comparison of mean directions or mean axes, The Annals of Statistics 26 (2) (1998) 472-493.

[29] H. B. Nielsen, Damping parameter in Marquardt's method, Tech. Rep. IMM-REP-1999-05, IMM, Department of Mathematical Modelling, Technical University of Denmark, Lyngby, Denmark (Apr. 1999).

[30] Microsoft, Kinect hardware, https://dev.windows.com/en-us/kinect/ hardware, accessed: 2015-10-08.

[31] LORD MicroStrain, 3DM-GX3-25, http://www.microstrain.com/ inertial/3DM-GX3-25, accessed: 2015-10-08.

[32] P. Fankhauser, M. Bloesch, D. Rodriguez, R. Kaestner, M. Hutter, R. Siegwart, Kinect v2 for mobile robot navigation: Evaluation and modeling, in: Proceedings of the 17th International Conference on Advanced Robotics, Istanbul, Turkey, 2015, pp. 388-394.

[33] LORD MicroStrain, 3DM-GX3-25 Miniature Attitude Heading Reference System, 8400-0033 Rev. 003 (2014).

[34] Renishaw plc, Quarryman Pro LR Hardware Manual, H-5915-8501-02-A (Dec. 2014).

750 [35] Rocscience, Dips, v. 6.0, Toronto, ON, 2015. 